\title{
Structure of hard-hypersphere fluids in odd dimensions
}

\author{
René D. Rohrmann $*$ and Andrés Santo: \\ Departamento de Física, Universidad de Extremadura, E-06071 Badajoz, Spain
}

(Dated: November 21, 2018)

\begin{abstract}
The structural properties of single component fluids of hard hyperspheres in odd space dimensionalities $d$ are studied with an analytical approximation method that generalizes the Rational Function Approximation earlier introduced in the study of hard-sphere fluids [S. B. Yuste and A. Santos, Phys. Rev. A 43, 5418 (1991)]. The theory makes use of the exact form of the radial distribution function to first order in density and extends it to finite density by assuming a rational form for a function defined in Laplace space, the coefficients being determined by simple physical requirements. Fourier transform in terms of reverse Bessel polynomials constitute the mathematical framework of this approximation, from which an analytical expression for the static structure factor is obtained. In its most elementary form, the method recovers the solution of the Percus-Yevick closure to the Ornstein-Zernike equation for hyperspheres at odd dimension. The present formalism allows one to go beyond by yielding solutions with thermodynamic consistency between the virial and compressibility routes to any desired equation of state. Excellent agreement with available computer simulation data at $d=5$ and $d=7$ is obtained.

PACS numbers: 61.20.Gy, 61.20.Ne, 05.20.Jj, 51.30.+i
\end{abstract}

\section{INTRODUCTION}

Fluid systems made of hard bodies constitute simple models in which impenetrable particles interact solely through hard-core repulsions. Despite their simplicity, hard-sphere systems are commonly used as reference models to obtain accurate descriptions of real substances (simple fluids, colloidal suspensions, granular media, and glasses) over a wide range of state conditions [1, 2, 3]. In particular, hard-sphere systems exhibit typical liquidlike phenomena, such as a first-order freezing transition [4, [5, 6] and metastable glass states [7].

Hard-hypersphere fluids (where the interaction potential is infinite when two hyperspheres overlap and zero otherwise) are the natural extension of hard spheres to arbitrary dimensions $d$. Such systems have attracted an everlasting attention of many researchers $6,8,8,9,10,11$, $12,13,14,15,16,17,18,19,20,21,22,23,24,25,26,27$, $28,29,30,31,32,33,34,35,36,37,38,39,40,41,42,43$, 44, 45, 46, 47]. The main reason is twofold. First, studies of hard particles in high dimensions may reveal general behaviors of the equation of state (EOS), radius of convergence of the virial series, phase transitions, and fluid structure that can help to understand the corresponding properties in real fluids. Second, hypersphere systems provide well defined and very demanding test models for theoretical approximations to many-body problems.

The thermodynamic and structural properties of $d$ sphere fluids in high dimensions have been examined by computer simulations [6, 29, 31, 36, 37, 39, 40, 41, 44, 45].

*Electronic address: rohr@oac.uncor.edu Permanent address: Observatorio Astronómico, Universidad Nacional de Córdoba, Laprida 854, X5000BGR Córdoba, Argentina

${ }^{\dagger}$ Electronic address:

URL: http://www.unex.es/fisteor/andres
At a theoretical level, a number of virial coefficients have been evaluated [9, 10, 32, 33, 34, 38], the asymptotic properties in the limit of infinitely many dimensions have been investigated $12,15,22,23,26,35$, several approximate EOS have been proposed 16, 17, 18, 19, 20, 21, 25, 27, 28], and scaled-particle and density-functional methods have been applied to the fluid-solid phase transition [24, 30]. Regarding the structural properties, the PercusYevick (PY) closure to the Ornstein-Zernike (OZ) relation has been proven to be exactly solvable at $d=$ odd [8, 11], the solution having been worked out at $d=5$ [8] and $d=731,43$ (apart from the classical cases of $d=1$ [48] and $d=3[49,[50])$, and overlap volume function representations have been proposed [13, 14]. Additionally, special interest has focused on the packing problem and the formation of jammed structures [41, 42, 46, 47, 51].

The primary aim of this paper is to introduce an analytical method for the study of hard-particle fluids in Euclidean spaces of arbitrary odd dimension. Specifically, we derive an expression for a key function directly related to the static structure factor $S(k)$ of the $d$-sphere fluid, from which all other structural and thermodynamic properties can be expressed. Our technique is based on the Rational Function Approximation (RFA) method, originally developed for three-dimensional hard spheres [52] and applied to a wide variety of problems [53], including hard-sphere mixtures [54], sticky hard spheres [55, 56, 57], square-well fluids [58, 59], and penetrable spheres [60]. In the RFA approach developed in this paper we define a Laplace-space functional $G(s)$ of the radial distribution function $g(r)$ that allows one to obtain $S(k)$ in a simple way. By making use of the exact form of $g(r)$ to first order in density, a function $\Psi(s)$ directly related to $G(s)$ is approximated by a Padé approximant, its coefficients being constrained by basic physical conditions arising from the small wavenumber behavior of $S(k)$. The simplest implementation of the approach, i.e., 
the one with an equal number of coefficients and constraints, turns out to coincide with the PY solution. The next extension contains two extra coefficients that are fitted to reproduce any desired EOS in a thermodynamically consistent way.

The paper is organized as follows. Section [I] summarizes the basic physical tools involved in this study and describes the application of reverse Bessel polynomials in the evaluation of Fourier transforms in odd dimensional space. An explicit expression for the overlap volume between two hyperspheres, which plays a prominent role in the generalization of the RFA method, is derived in Sec. III Section IV is devoted to the asymptotic expressions of $S(k)$ and $G(s)$ for long wavenumber, for short wavenumber, and for low densities. The generalization of the RFA approach to arbitrary odd dimension is presented in Sec. $\mathrm{V}$ and the corresponding evaluation of the direct correlation function $c(r)$ is given in Sec. VI Section VII shows explicit results for fluids in dimensions $d \leq 11$ and comparisons with available computer simulations for $d=5$ and $d=7$. The paper is closed with some concluding remarks in Sec. VIII.

\section{FRAMEWORK}

\section{A. Definitions}

The structure of a fluid is typically studied in terms of the radial distribution function, $g(r)$, and a closely related function, the structure factor $S(k)$, given by

$$
S(k)=1+\rho \widehat{h}(k) .
$$

Here $k$ is the wavenumber, $\rho$ is the density, and $\widehat{h}(k)$ is the Fourier transform of the total correlation function,

$$
h(r)=g(r)-1 .
$$

While $g(r)$ gives the relative probability of finding a particle located a distance $r$ from another particle located at the origin, $S(k)$ is proportional to the scattered intensity of radiation from the fluid and thus is obtainable from scattering experiments. An additional useful quantity to describe the fluid structure is the direct correlation function $c(r)$, which is defined through the $\mathrm{OZ}$ relation. In Fourier space it reads

$$
\widehat{c}(k)=\frac{\widehat{h}(k)}{1+\rho \widehat{h}(k)}=\frac{1}{\rho}\left[1-\frac{1}{S(k)}\right] .
$$

The thermodynamics of hard $d$-sphere fluids can be fully accounted for by the compressibility factor $Z \equiv$ $p / \rho k_{B} T$ (dimensionless combination of pressure $p$, density $\rho$, and temperature $T$ ), which can be evaluated in turn from the contact value of the radial distribution function, $g\left(\sigma^{+}\right)$,

$$
Z=1+2^{d-1} \eta g\left(\sigma^{+}\right)
$$

where $\eta$ is the fraction of the total volume occupied by the $d$-spheres (or packing fraction) and $\sigma$ the diameter of a particle. The general relation between $\rho$ and $\eta$ reads

$$
\eta=v_{d} \rho \sigma^{d}, \quad v_{d}=\frac{(\pi / 4)^{d / 2}}{\Gamma(1+d / 2)},
$$

where $v_{d}$ is the volume of a $d$-dimensional sphere of unit diameter. In odd dimensions,

$$
v_{d}=\frac{(\pi / 2)^{(d-1) / 2}}{d ! !} .
$$

Furthermore, the structure factor is related to thermodynamics through the isothermal susceptibility as follows,

$$
\chi \equiv k_{B} T\left(\frac{\partial \rho}{\partial p}\right)_{T}=S(0) .
$$

The relation between the isothermal susceptibility and the compressibility factor is given by

$$
\chi^{-1}=\frac{\mathrm{d}}{\mathrm{d} \eta}(\eta Z) .
$$

This equation can be used to impose thermodynamic consistency between the virial and compressibility routes to the EOS.

For hard-particle systems in equilibrium, the structural properties considered here are athermal, i.e., the temperature does not play any relevant role. Moreover, the thermodynamic state can be characterized by a variable alone, i.e., the density, the pressure, or any related variable (e.g., $\eta, Z, \chi)$.

\section{B. Low density expansions}

We consider briefly some exact low density results which will be included in the analytical theory presented in this paper. We start from the following general relationship

$$
g(r)=[1+f(r)] y(r),
$$

where $y(r)$ is the cavity function and $f(r)$ is the Mayer function, which in the case of hard $d$-spheres reads

$$
f(r)=\left\{\begin{array}{r}
-1, \quad r<\sigma \\
0, \quad r>\sigma .
\end{array}\right.
$$

The low density behavior of $g(r)$ can be derived from the virial expansion of the cavity function,

$$
y(r)=1+y_{1}(r) \rho+y_{2}(r) \rho^{2}+\cdots,
$$

where the functions $y_{n}(r)$ are represented by cluster diagrams [1, 2]. In particular, the first-order contribution to the cavity function is

$$
y_{1}(r)=\int \mathrm{d} \mathbf{r}^{\prime} f\left(r^{\prime}\right) f\left(\left|\mathbf{r}-\mathbf{r}^{\prime}\right|\right) .
$$


It is worth noting that, because of Eq. (2.10), $y_{1}(r)$ represents the intersection volumen of two identical $d$ dimensional spheres of radius $\sigma$ whose centers are separated by a distance $r$ [61].

With the preceding expressions, the lowest order terms in the $\eta$-expansion of the radial distribution function,

$$
g(r)=g_{0}(r)+g_{1}(r) \eta+O\left(\eta^{2}\right)
$$

are

$$
\begin{aligned}
& g_{0}(r)=1+f(r)=\Theta(r-\sigma), \\
& g_{1}(r)=\frac{\sigma^{-d}}{v_{d}}[1+f(r)] y_{1}(r),
\end{aligned}
$$

where $\Theta(x)$ is the step function $[\Theta(x)=1$ if $x>1$ and zero otherwise]. The factor $1+f(r)$ prevents any pair of particle centers from getting closer than a distance $\sigma$.

Fourier transformation of Eq. (2.15) gives

$$
\widehat{g}_{1}(k)=\frac{\sigma^{-d}}{v_{d}}\left[\widehat{y}_{1}(k)+\frac{1}{(2 \pi)^{d}} \int \mathrm{d} \mathbf{k}^{\prime} \widehat{y}_{1}\left(k^{\prime}\right) \widehat{f}\left(\left|\mathbf{k}-\mathbf{k}^{\prime}\right|\right)\right],
$$

where, by application of the convolution theorem in (2.12),

$$
\widehat{y}_{1}(k)=[\widehat{f}(k)]^{2} .
$$

\section{Fourier transform in odd dimensions}

The Fourier transform of an absolutely integrable function $\xi(\mathbf{r})$ in $d$ dimensions is defined by

$$
\widehat{\xi}(\mathbf{k})=\int \mathrm{d} \mathbf{r} \xi(\mathbf{r}) e^{-i \mathbf{k} \cdot \mathbf{r}}
$$

and the associated inverse operation is given by

$$
\xi(\mathbf{r})=\frac{1}{(2 \pi)^{d}} \int \mathrm{d} \mathbf{k} \widehat{\xi}(\mathbf{k}) e^{i \mathbf{k} \cdot \mathbf{r}},
$$

where $\mathbf{k}$ is the wave vector. It is proven in Appendix A that, if the function $\xi(\mathbf{r})=\xi(r)$ depends only on the magnitude $r=|\mathbf{r}|$ of the vector $\mathbf{r}$ and $d=$ odd, then the $d$-dimensional direct and inverse Fourier transforms (2.18) and (2.19) can be expressed as

$$
\begin{gathered}
\widehat{\xi}(k)=2 \frac{(2 \pi)^{(d-1) / 2}}{k^{d-2}} \operatorname{Im}\left\{\mathcal{F}_{n}[\xi(r)](-i k)\right\}, \\
\xi(r)=2 \frac{(2 \pi)^{-(d+1) / 2}}{r^{d-2}} \operatorname{Im}\left\{\mathcal{F}_{n}[\widehat{\xi}(k)](-i r)\right\},
\end{gathered}
$$

respectively. Here, $\operatorname{Im}(z)$ denotes the imaginary part of $z, n$ is an integer related to $d$ by

$$
n \equiv \frac{d-3}{2}, \quad d=2 n+3,
$$

TABLE I: Reverse Bessel polynomials of degree less than seven.

\begin{tabular}{ll}
\hline \hline$n$ & $\theta_{n}(t)$ \\
\hline 0 & 1 \\
1 & $1+t$ \\
2 & $3+3 t+t^{2}$ \\
3 & $15+15 t+6 t^{2}+t^{3}$ \\
4 & $105+105 t+45 t^{2}+10 t^{3}+t^{4}$ \\
5 & $945+945 t+420 t^{2}+105 t^{3}+15 t^{4}+t^{5}$ \\
6 & $10395+10395 t+4725 t^{2}+1260 t^{3}+210 t^{4}+21 t^{5}+t^{6}$ \\
\hline \hline
\end{tabular}

and $\mathcal{F}_{n}[\xi(x)](s)$ is a functional of $\xi(x)$ defined by

$$
\mathcal{F}_{n}[\xi(x)](s) \equiv \int_{0}^{\infty} \mathrm{d} x x \xi(x) \theta_{n}(s x) e^{-s x},
$$

where the function $\theta_{n}(t)$ is the so-called reverse Bessel polynomial of degree $n[62,63,64,65$, 66], whose expression is

$$
\theta_{n}(t)=\sum_{j=0}^{n} \omega_{n, j} t^{j}, \quad \omega_{n, j}=\frac{(2 n-j) !}{2^{n-j}(n-j) ! j !} .
$$

Some of the properties of $\theta_{n}(t)$ are summarized in Appendix A. Table I provides the polynomials $\theta_{n}(t)$ of degree less than seven.

Two useful applications of Eq. (2.23) correspond to $\xi(x)=1$ and to the step function $\xi(x)=\Theta(x-1)$. With the help of (A5) and (2.24) one finds

$$
\begin{gathered}
\mathcal{F}_{n}[1](s)=\frac{\theta_{n+1}(0)}{s^{2}}=\frac{(2 n+1) ! !}{s^{2}}, \\
\mathcal{F}_{n}[\Theta(x-1)](s)=\frac{\theta_{n+1}(s) e^{-s}}{s^{2}} .
\end{gathered}
$$

Henceforth we will indistinctly use $d$ and $n=(d-$ $3) / 2$ in the remainder of the paper. Except for a few exceptions, we will generally follow the rule of employing $n$ in subscripts and $d$ in exponents.

\section{THE TWO-HYPERSPHERE OVERLAP VOLUME}

The intersection volume of two $d$-spheres whose centers are separated a distance $r$ is a key quantity in the study of hard systems [42, 67]. Apart from its geometrical interest, it yields the cavity function to first order in density, $y_{1}(r)$, as mentioned below Eq. (2.12). Some expressions of $y_{1}(r)$ in terms of special functions and recurrence relations can be found in the literature [14, 16, 68], and an explicit expression has been recently derived by Torquato and Stillinger [42]. In this Section we use the representation (2.21) for $d=2 n+3=$ odd to provide an alternative analytical expression of $y_{1}(r)$. 
Taking into account that $f(r)=\Theta(r-1)-1$, where henceforth we take $\sigma$ as the length unit (i.e., $\sigma=1$ ) and making use of Eqs. (2.20), (2.25), and (2.26), one has [69]

$$
\widehat{f}(k)=\frac{(2 \pi)^{(d-1) / 2}}{k^{d}} i\left[\theta_{n+1}(-i k) e^{i k}-\theta_{n+1}(i k) e^{-i k}\right] .
$$

From the property (A10) it is easy to prove that, as expected

$$
\lim _{k \rightarrow 0} \widehat{f}(k)=-\frac{2(2 \pi)^{(d-1) / 2}}{d ! !}=-2^{d} v_{d} .
$$
by

The contribution $y_{1}(r)$ to the cavity function is given

$$
y_{1}(r)=\frac{(2 \pi)^{-(d+1) / 2}}{r^{d-2}} i \int_{-\infty}^{\infty} \mathrm{d} k k \widehat{y}_{1}(k) \theta_{n}(i k r) e^{-i k r},
$$

where, according to (2.17) and (3.1),

$$
\begin{aligned}
\widehat{y}_{1}(k)= & \frac{(2 \pi)^{d-1}}{k^{2 d}}\left[2 \theta_{n+1}(i k) \theta_{n+1}(-i k)\right. \\
& \left.-\theta_{n+1}^{2}(i k) e^{-2 i k}-\theta_{n+1}^{2}(-i k) e^{2 i k}\right] .
\end{aligned}
$$

Since $\widehat{y}_{1}(0)=\left(2^{d} v_{d}\right)^{2}=$ finite, the integrand in (3.3) is regular along the integration interval and so we can consider an integration path in the complex plane from $k=-\infty$ to $k=+\infty$ that goes round the point $k=0$ from below. The integral in Eq. (3.3) decomposes into three contributions with integrands headed by $e^{-i k r}, e^{-i k(r+2)}$ and $e^{-i k(r-2)}$, respectively. If $r>2$, we can close the path with a lower half-circle of infinite radius, so that the three contributions vanish. If $0<r<2$, however, the path in the third integral must be closed with an upper half-circle and the residue theorem yields a nonzero value. Therefore, for positive $r$ one has

$$
y_{1}(r)=\frac{(2 \pi)^{(d-1) / 2}}{r^{d-2}} R_{4 n+4}(r) \Theta(2-r),
$$

where the residue

$$
R_{4 n+4}(r)=\operatorname{Res}_{k=0}\left[k^{-(2 d-1)} \theta_{n+1}^{2}(-i k) \theta_{n}(i k r) e^{i k(2-r)}\right]
$$

is given by the term of order $2 d-2=4 n+4$ in the $k$-expansion of $\theta_{n+1}^{2}(-i k) \theta_{n}(i k r) e^{i k(2-r)}$, i.e.

$$
R_{4 n+4}(r)=\left.\frac{1}{(4 n+4) !} \partial_{t}^{4 n+4} \theta_{n+1}^{2}(-t) \theta_{n}(r t) e^{(2-r) t}\right|_{t=0}
$$

where we have made the change $t=i k$. Equation (3.7) implies that $R_{4 n+4}(r)$ is a polynomial of degree $4 n+4$, as indicated by the notation. Since $\theta_{n+1}^{2}(-t) \theta_{n}(r t)$ is a polynomial of degree $3 n+2$ in $t$, the Taylor expansion of the exponential factor contributes to $R_{4 n+4}(r)$ with factors $(2-r)^{j}$ with $j \geq n+2$. Therefore, $R_{4 n+4}(r)$ factorizes into $(2-r)^{n+2}$ times a polynomial of degree $3 n+2$. According to Eq. (3.5), the latter polynomial starts with $r^{d-2}=r^{2 n+1}$ because $y_{1}(r)$ must remain finite when $r \rightarrow 0$. From this analysis, we obtain

$$
R_{4 n+4}(r)=r^{2 n+1}(2-r)^{n+2} P_{n+1}(r),
$$

where $P_{n+1}(r)$ is a polynomial of degree $n+1$. An explicit expression of $P_{n+1}(r)$ is given in Appendix B. We finally obtain the first-order contribution to the cavity function for hard-sphere fluids in odd dimensions or, equivalently, the overlap volume of two hyperspheres of radius $\sigma=1$ with centers separated a distance $r$ 61],

$$
y_{1}(r)=\Theta(2-r)(2 \pi)^{(d-1) / 2}(2-r)^{(d+1) / 2} P_{n+1}(r) .
$$

Notice that $y_{1}(0)$ is equivalent to the volume of one hypersphere the radius $\sigma=1$, i.e., $y_{1}(0)=2^{d} v_{d}$. Moreover, setting $r=1$ in Eq. (B5) yields

$$
\frac{y_{1}(1)}{y_{1}(0)}=1-\frac{(2 n+3) ! !}{2^{n+2}} \sum_{j=0}^{n+1} \frac{(-4)^{-j}}{(2 j+1) j !(n+1-j) !} .
$$

This result provides a simple expression for the third virial coefficient $B_{3}$ of hard $d$-sphere systems in odd dimensions. From the virial expansion

$$
Z=1+\sum_{l=2}^{\infty} B_{l} \rho^{l-1}
$$

and Eqs. (2.4) and (2.11) one has $B_{2}=2^{d-1} v_{d}$ and $B_{3}=$ $2^{d-1} v_{d} y_{1}(1)$. Therefore,

$$
\frac{B_{3}}{B_{2}^{2}}=2 \frac{y_{1}(1)}{y_{1}(0)}
$$

\section{ASYMPTOTIC BEHAVIORS OF THE STRUCTURE FACTOR}

In this Section we examine the asymptotic long wavenumber, short wavenumber, and low density behaviors of the structure factor and of a closely associated function, $G(s)$, which will play a central role in the next Sections. We define $G(s)$ as the functional $\mathcal{F}_{n}$, introduced in (2.23), of the radial distribution function,

$$
G(s) \equiv \mathcal{F}_{n}[g(r)](s)=\int_{0}^{\infty} \mathrm{d} r r g(r) \theta_{n}(s r) e^{-s r} .
$$

We have found that this is the optimal generalization to any odd dimension of the Laplace transform $G(s)=$ $\mathcal{L}[\mathrm{rg}(r)](s)$ used in Ref. [52] in the study of hard spheres $(d=3, n=0)$. With (2.2) and (2.25), we note that

$$
G(s)=\frac{(d-2) ! !}{s^{2}}+\int_{0}^{\infty} \mathrm{d} r r h(r) \theta_{n}(s r) e^{-s r} .
$$

Hence, using (2.1) and (2.20), the structure factor is given by

$$
S(k)=1+\rho \frac{(2 \pi)^{(d-1) / 2}}{k^{d-2}} i[G(i k)-G(-i k)] .
$$




\section{A. Long and short wavenumber limits}

We shall now derive general conditions that the functions $G(s)$ and $S(k)$ must satisfy. First, one can easily obtain an asymptotic expression of $G(s)$ for large $s$ by replacing $r g(r)=\Theta(r-1)\left[g\left(1^{+}\right)+O(r-1)\right]$ and $\theta_{n}(s r)=s^{n} r^{n}\left[1+O\left(s^{-1}\right)\right]$ in (4.1). The result is

$$
G(s)=g\left(1^{+}\right) s^{(d-5) / 2} e^{-s}\left[1+O\left(s^{-1}\right)\right],
$$

i.e.

$$
\lim _{s \rightarrow \infty} s^{(5-d) / 2} e^{s} G(s)=g\left(1^{+}\right)
$$

It follows from Eqs. (4.3) and (4.5) that, at long wavenumber, $S(k)$ adopts the form

$$
S(k) \approx 1+\frac{2^{d} d ! !}{k^{(d+1) / 2}} \eta g\left(1^{+}\right) \sin \left[k+\frac{\pi}{4}(5-d)\right] .
$$

Therefore, the structure factor for long $k$ oscillates with an amplitude proportional to the contact value $g\left(1^{+}\right)$, an envelope decaying as $k^{-(d+1) / 2}$, and a phase shift equal to $\pi(5-d) / 4$.

On the other hand, the asymptotic form of $G(s)$ for small $s$ can be derived from (4.2) by using Eq. (2.24) and the Taylor expansion of $e^{-s r}$. The result is

$$
G(s)=\frac{(d-2) ! !}{s^{2}}+\sum_{j=0}^{\infty} \alpha_{n, j} H_{j+1} s^{j}
$$

where

$$
H_{j} \equiv \int_{0}^{\infty} \mathrm{d} r r^{j} h(r)
$$

is the $j$ th moment of the total correlation function and the numerical coefficients $\alpha_{n, j}$ are given by

$$
\alpha_{n, j}=\sum_{l=0}^{\min (n, j)} \frac{(-1)^{j-l}}{(j-l) !} \omega_{n, l}
$$

One can verify that the first $n$ coefficients $\alpha_{n, j}$ with $j=$ $2 m+1=$ odd vanish, i.e.,

$$
\alpha_{n, 2 m+1}=0, \quad m=0,1, \ldots, n-1 .
$$

Therefore,

$$
\begin{aligned}
G(s)= & \frac{(d-2) ! !}{s^{2}}+\sum_{m=0}^{\infty} \alpha_{n, 2 m} H_{2 m+1} s^{2 m} \\
& +\sum_{m=n}^{\infty} \alpha_{n, 2 m+1} H_{2 m+2} s^{2 m+1} .
\end{aligned}
$$

The property (4.10) is essential to guarantee that $S(k)$ remains bounded at the limit of zero wavenumber and, consequently, the isothermal susceptibility takes finite values. Thus, inserting Eq. (4.11) into Eq. (4.3), we get the Taylor expansion of $S(k)$ as

$$
S(k)=1-2^{d} d ! ! \eta \sum_{m=n}^{\infty}(-1)^{m} \alpha_{n, 2 m+1} H_{2 m+2} k^{2(m-n)}
$$

Application of Eq. (2.7) provides a direct relationship between the $(d-1)$ th moment of the total correlation function and the isothermal susceptibility, namely

$$
\chi=1+2^{d} d \eta H_{d-1},
$$

where use has been made of the property

$$
\alpha_{n, 2 n+1}=\frac{(-1)^{n+1}}{(2 n+1) ! !}
$$

\section{B. Low density expansion}

The series expansion of the radial distribution function in terms of the packing fraction $\eta$, Eq. (2.13), leads to a similar expansion for the function $G(s)$,

$$
G(s)=G_{0}(s)+G_{1}(s) \eta+O\left(\eta^{2}\right),
$$

with $G_{j}(s)=\mathcal{F}_{n}\left[g_{j}(r)\right](s)$. The zeroth order term is derived from Eqs. (2.14) and (2.26),

$$
G_{0}(s)=s^{-2} \theta_{n+1}(s) e^{-s} .
$$

Next, since, $g(r)=\Theta(r-1) y(r)$ and $y_{1}(r)$ vanishes for $r \geq 2$, one can write

$$
G_{1}(s)=\frac{1}{v_{d}} \int_{1}^{2} \mathrm{~d} r r y_{1}(r) \theta_{n}(s r) e^{-s r} .
$$

It is proven in Appendix $\mathrm{C}$ that

$$
G_{1}(s)=\frac{\lambda}{s^{d-2}} G_{0}^{2}(s)+\frac{e^{-s}}{s^{d+2}} Q_{3 n+4}(s),
$$

where

$$
\lambda \equiv(-1)^{(d-1) / 2} 2^{d-1} d ! !
$$

and $Q_{3 n+4}(s)$ is a polynomial of degree $3 n+4=(3 d-$ 1)/2. Explicit expressions of the first few polynomials $Q_{3 n+4}(s)$ are given in Table II]

\section{RATIONAL FUNCTION APPROXIMATION}

The results presented in the preceding Sections are exact. In this Section, we propose the extension to hyperspheres in arbitrary odd dimensions of the RFA, originally introduced in the study of three-dimensional hardsphere systems [52, 53, 70]. The main steps in the RFA can be summarized as follows: (i) a functional $G(s)$ of the 
TABLE II: Polynomials $Q_{3 n+4}(s)$ associated with dimensions $d=3,5,7$, and 9 .

\begin{tabular}{ll}
\hline \hline$n$ & $Q_{3 n+4}(s)$ \\
\hline 0 & $12+12 s-6 s^{2}-2 s^{3}+(5 / 2) s^{4}$ \\
1 & $-2160-2160 s-360 s^{2}+360 s^{3}+30 s^{4}-42 s^{5}+3 s^{6}+(53 / 8) s^{7}$ \\
2 & $1512000+1512000 s+453600 s^{2}-50400 s^{3}-47880 s^{4}+2520 s^{5}+2940 s^{6}-300 s^{7}-(285 / 2) s^{8}$ \\
& $+(789 / 16) s^{9}+(289 / 16) s^{10}$ \\
3 & $-2667168000-2667168000 s-952560000 s^{2}-63504000 s^{3}+46720800 s^{4}+8618400 s^{5}-1738800 s^{6}$ \\
& $-378000 s^{7}+69930 s^{8}+11130 s^{9}-3045 s^{10}-(585 / 128) s^{11}+(38615 / 128) s^{12}+(6413 / 128) s^{13}$ \\
\hline \hline
\end{tabular}

radial distribution function $g(r)$ is defined by a suitable Laplace transformation such that $G(s)$ is simply related to the structure factor $S(k)$; (ii) using as a guide the low density form of $G(s)$, an auxiliary function $\Psi(s)$ is introduced; (iii) the unknown function $\Psi(s)$ is approximated by a rational function (or Padé approximant), the degree difference between the numerator and denominator polynomials being dictated by the exact large- $s$ behavior of $G(s)$; (iv) finally, the coefficients of the rational form for $\Psi(s)$ are determined by requiring consistency with the exact small- $s$ behavior of $G(s)$.

According to Eq. (4.3), the first step described above is accomplished by the functional $G(s)$ defined by Eq. (4.1). Its large- $s$ and small- $s$ behaviors are given by Eqs. (4.5) and (4.11), respectively. In order to continue with the step (ii), let us rewrite Eq. (4.15) as

$$
G(s)=\frac{s^{d-2}}{\Psi_{0}(s) e^{s}}+\frac{s^{d-2}}{\Psi_{0}^{2}(s) e^{2 s}}\left[\lambda-\Psi_{1}(s) e^{s}\right] \eta+O\left(\eta^{2}\right),
$$

where, according to Eqs. (4.16) and (4.18),

$$
\begin{gathered}
\Psi_{0}(s)=\frac{s^{d}}{\theta_{n+1}(s)}, \\
\Psi_{1}(s)=-\frac{Q_{3 n+4}(s)}{\theta_{n+1}^{2}(s)^{2}} .
\end{gathered}
$$

This suggests the introduction of the auxiliary function $\Psi(s)$ through

$$
G(s)=\frac{s^{d-2}}{\Psi(s) e^{s}-\lambda \eta}
$$

so that

$$
\Psi(s)=\Psi_{0}(s)+\Psi_{1}(s) \eta+O\left(\eta^{2}\right) .
$$

The large- $s$ and small- $s$ conditions (4.5) and (4.11) imply that

$$
\begin{gathered}
\lim _{s \rightarrow \infty} s^{-(d+1) / 2} \Psi(s)=\frac{1}{g\left(1^{+}\right)} \\
\left.\partial_{s}^{j} \Psi(s) e^{s}\right|_{s=0}= \begin{cases}\lambda \eta, & j=0 \\
0, & 1 \leq j \leq d-1, \\
d ! /(d-2) ! !, & j=d, \\
0, & j=d+1+2 m, \\
& m=0, \ldots, n,\end{cases}
\end{gathered}
$$

respectively, where in Eq. (5.7) we have taken into account Eq. (4.10).

Thus far, Eqs. (5.1) - 5.7) are exact. Now we follow step (iii) and approximate the auxiliary function $\Psi(s)$ by a rational form (or Padé approximant),

$\Psi(s)=\frac{B_{N}(s)}{A_{M}(s)}, \quad A_{M}(s) \equiv \sum_{j=0}^{M} a_{j} s^{j}, \quad B_{N}(s) \equiv \sum_{j=0}^{N} b_{j} s^{j}$

Note that the choice of a rational form for $\Psi(s)$ is compatible with the exact requirements (5.2), (5.3), (5.6), and (5.7). The combination of Eqs. (5.4) and (5.8) constitute a simple approximation of $G(s)$ that will be made consistent with the basic physical requirements outlined in Section IV. To begin with, we note that the condition (5.6) fixes the relation between the degrees of the polynomials $A_{M}(s)$ and $B_{N}(s)$, namely

$$
N=M+\frac{d+1}{2} .
$$

In fact, the ratio of the highest coefficients $a_{M}$ and $b_{N}$ directly gives the contact value of the pair distribution function:

$$
g\left(1^{+}\right)=\frac{a_{M}}{b_{N}}
$$

To close the RFA proposal (5.8) we need to determine the coefficients $\left\{a_{j}\right\}$ and $\left\{b_{j}\right\}$. Since one of them can be arbitrarily chosen, the number of independent unknowns is $N+M+1$. Following the step (iv), we resort to the exact small-s behavior (5.7), which imposes $d+n+2=$ $3 n+5$ constraints. Therefore, in order to make the problem solvable, one must have $N+M \geq d+n+1=3 n+4$. In view of Eq. (5.9), this implies that

$$
N \geq d, \quad M \geq n+1=\frac{d-1}{2} .
$$

The constraints stemming from Eq. (5.7) or, equivalently, Eq. (4.11) are worked out in Appendix D.

\section{A. Low density expansion}

It is worthwhile noting that the lower bounds (5.11) can also be derived by requiring consistency of the RFA 
form (5.8) with the exact zeroth order term, Eq. (5.2), in a density expansion. In the approximation with the least number of unknowns, i.e., with

$$
N=d, \quad M=n+1,
$$

Eq. (5.2) implies that

$$
\left.\frac{b_{j}}{b_{d}}\right|_{\eta=0}=\delta_{j, d},\left.\quad \frac{a_{j}}{b_{d}}\right|_{\eta=0}=\omega_{n+1, j},
$$

where $\delta_{j, d}$ is Kronecker's delta. As mentioned before, one of the coefficients in (5.8) can be given a constant, arbitrary non-zero value. Of course, this choice will not have any consequence on the physical results derived from (5.4), but an appropriate one may simplify the algebra involved in the further development below. In this sense, in view of (5.13), two adequate alternative choices are either

$$
a_{0} \equiv \omega_{n+1,0}=(d-2) ! !
$$

or

$$
b_{d} \equiv 1 \text {. }
$$

Henceforth we will adopt (5.14). With the assumption (5.12) and the choice (5.14), it is seen from Eq. (5.3) that the derivatives $\left.a_{j}^{\prime} \equiv \partial_{\eta} a_{j}\right|_{\eta=0}$ and $\left.b_{j}^{\prime} \equiv \partial_{\eta} b_{j}\right|_{\eta=0}$ obey the following equations:

$$
b_{j}^{\prime}=-\frac{1}{(d-2) ! !}\left[q_{3 n+4, j}+\sum_{m=\max (0, j-M)}^{j-1} \omega_{n+1, j-m} b_{m}^{\prime}\right],
$$

for $0 \leq j \leq N=d$, and

$$
a_{j}^{\prime}=q_{3 n+4, N+j}+\sum_{m=j}^{M} \omega_{n+1, m} b_{N+j-m}^{\prime},
$$

for $1 \leq j \leq M=n+1$, where $q_{3 n+4, j}$ is the $j$ th coefficient of the polynomial $Q_{3 n+4}(s)$. Application of (5.16) allows one to obtain recursively all the quantities $b_{j}^{\prime}$, which can then be used in the evaluation of $a_{j}^{\prime}$ with the help of (5.17).

In conclusion, the analytical form provided by Eqs. (5.4) and (5.8) exactly reproduces the zeroth- and firstorder terms in density of $G(s)$ when suitable values of the Padé coefficients and their derivatives are used. In the next subsection, we carry out the extension of this representation to arbitrary densities.

\section{B. Standard approximation}

Let us consider the RFA form (5.8) when the number of unknowns $(N+M+1)$ equals the number of constraints $(3 n+5)$. Taking into account Eq. (5.9), this corresponds to the choice (5.12). We will refer to this case as the standard RFA, i.e., the one in which all the Padé coefficients are determined from the basic constraints and so no free parameters remain. As will be shown in Sec. VI this standard RFA turns out to provide the exact solution of the PY integral equation for odd dimensions [8, 11] by a completely different method.

For the case (5.12), Eq. (D7) expresses the coefficients $\left\{b_{j}\right\}$ in terms of the coefficients $\left\{a_{j}\right\}$. Insertion into Eqs. (5.4) and (5.8) allows one to rewrite $G(s)$ in the form

$$
G(s)=\frac{e^{-s}}{s^{2}} \frac{A_{n+1}(s)}{1+\lambda \eta \sum_{j=0}^{n+1} a_{j} \phi_{d-j}(s)},
$$

where we have called

$$
\phi_{m}(s) \equiv \frac{1}{s^{m}}\left[\sum_{j=0}^{m} \frac{(-s)^{j}}{j !}-e^{-s}\right] .
$$

Note that $\phi_{m}(0)=0$. Equation (5.18) assumes the normalization choice (5.14) [71].

In order to evaluate the $n+1$ coefficients $\left\{a_{j}, j=\right.$ $1, \ldots, n+1\}$ we can use the remaining constraints obtained from (D1). An equivalent procedure results from the substitution of (5.18) into (4.7), followed by a new power-series analysis. This latter method is simpler than the other one because the coefficients $\left\{b_{j}\right\}$ have already been eliminated. In this case, the analysis of powers yields

$$
D_{l}+\sum_{j=0}^{l-2} \gamma_{j} D_{l-2-j}=\widetilde{a}_{l},
$$

where we have called

$$
\widetilde{a}_{j} \equiv \frac{a_{j}}{(d-2) ! !}, \quad \gamma_{j} \equiv \frac{\alpha_{n, j} H_{j+1}}{(d-2) ! !} .
$$

The coefficients $D_{l}$ are linear combinations of the $\left\{a_{j}\right\}$ given by $D_{0}=1$ and

$$
D_{l}=\frac{1}{l !}-\lambda \eta \sum_{m=0}^{n+1} t_{d-m, l} a_{m}, \quad l \geq 1,
$$

where

$$
t_{m, j}=\sum_{l=1}^{j} \frac{(-1)^{l+m}}{(l+m) !(j-l) !}
$$

is the $j$ th coefficient in the power series expansion of $-e^{s} \phi_{m}(s)$. In Eq. (5.20) and in the remainder of this Section we have adopted the conventions $a_{j}=0$ if $j>n+1$ and $\sum_{j=0}^{m} \cdots=0$ if $m<0$.

Because of (4.10), we have

$$
\gamma_{1}=\gamma_{3}=\cdots=\gamma_{2 n-1}=0 .
$$




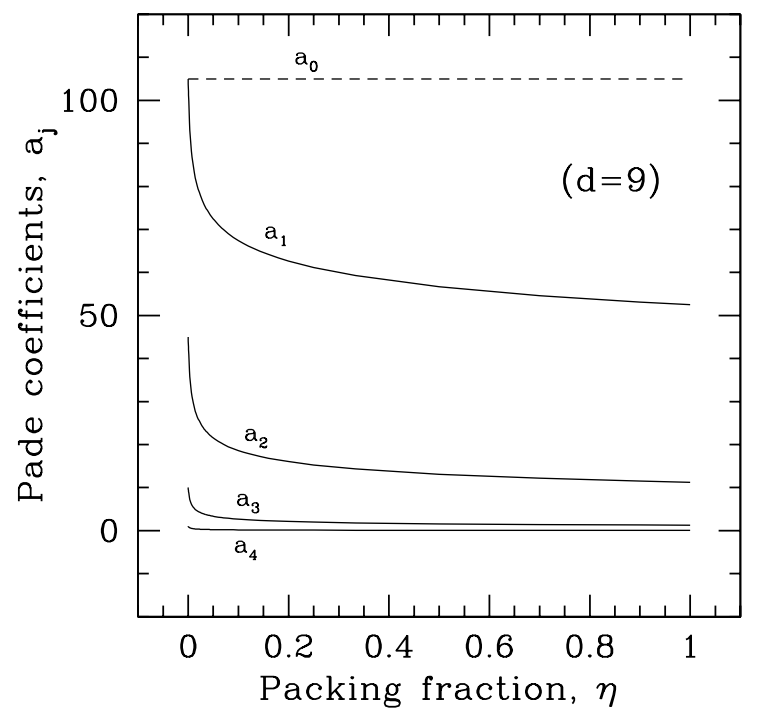

FIG. 1: Coefficients $a_{j}$, as a function of $\eta$, obtained from the standard RFA method for a fluid at $d=9$.

Therefore, Eq. (5.20) with $l=$ even $\leq 2 n+2$ can be used to express the quantities $\gamma_{2 m}$ with $0 \leq m \leq n$ in terms of the coefficients $a_{j}$ by means of the recursion relation

$\gamma_{2 m}=\widetilde{a}_{2 m+2}-D_{2 m+2}-\sum_{j=0}^{m-1} \gamma_{2 j} D_{2(m-j)}, \quad 0 \leq m \leq n$.

The parameters $\gamma_{0}, \gamma_{2}, \gamma_{4}, \gamma_{6}, \ldots$ are linear, quadratic, cubic, quartic, ... in the coefficients $\left\{a_{j}\right\}$. Next, Eq. (5.20) with $l=$ odd $\leq 2 n+1$ yields

$$
D_{2 m+1}+\sum_{j=0}^{m-1} \gamma_{2 j} D_{2(m-j)-1}=\widetilde{a}_{2 m+1}, \quad 0 \leq m \leq n .
$$

When the $\left\{\gamma_{2 m}\right\}$ obtained from Eq. (5.25) are inserted into Eq. (5.26) one gets a closed set of $n+1$ equations for $a_{1}, a_{2}, \ldots, a_{n+1}$. Therefore, the implementation of the standard RFA method reduces to solving a set of $n+1=(d-1) / 2$ algebraic equations, which become nonlinear for $n \geq 1$ or, equivalently, $d \geq 5$. In general, the number of mathematical solutions (including complex ones) is $2^{n}$. In the case of multiple solutions, we choose the solution which yields the correct low density limit given by (5.13). In this sense, it is important to note that the asymptotic behaviors of $a_{j}$ and $b_{j}$ as $\eta \rightarrow 0$ [Eqs. (5.13), (5.16), and (5.17)] are naturally included among the solutions of Eqs. (5.26). This is due to two reasons: (a) Eq. (4.11) is verified by the exact density expansion of $G(s)$ up to any order, in particular to zeroth and first orders [Eq. (4.15)]; (b) the number of constraints derived from (4.11) coincides with the least number of Padé coefficients required in the exact expansion of $G(s)$ up to first order in density.
The set of equations (5.26) can be analytically solved for $d=1,3,5$, and 7 , the solutions for $d=1,3$, and 5 being explicitly given in Appendix E, For $d=$ odd $\geq 9$, however, the solutions must be obtained numerically. By means of explicit evaluations in all the cases analyzed, we have found that the coefficients $\left\{a_{j}\right\}$ are finite for $0 \leq \eta \leq 1$ and adopt the following values at $\eta=1$ :

$$
\left.a_{j}\right|_{\eta=1}=\left.2^{-j} a_{j}\right|_{\eta=0}=\frac{\omega_{n+1, j}}{2^{j}} .
$$

Figure 1 illustrates the physical roots of Eq. (5.26) for a fluid of dimension $d=9(n=3)$ as functions of the packing fraction. It may be observed that, at the low density limit $(\eta \rightarrow 0)$, each $a_{j}$ tends to the corresponding coefficient $\omega_{4, j}$ of the reverse Bessel polynomial $\theta_{4}(t)$ (see Table I), as dictated by Eq. (5.13). These roots [except $a_{0}$ which is fixed by Eq. (5.14)] monotonically decrease as the density increases, reaching their minimum values given by Eq. (5.27) at $\eta=1$.

Once the coefficients $\left\{a_{j}\right\}$ are determined as functions of $\eta$, the structure factor of the system is given by Eqs. (4.3) and (5.18). The ansatz (5.8) thus provides an explicit expression for the structure factor to any finite density. Because the approximation to $G(s)$ is exact to first order in density, the structure factor thus obtained is exact to second order in density. In conclusion, the $4 n+5$ constraints derived from the small- $s$ behavior of $G(s)$, together with the zero-density conditions (5.13), allow us to completely characterize $S(k)$ for a hypersphere fluid in odd dimension in the standard RFA approach. Since $S(k)$ and $\widehat{h}(k)$ are directly related through Eq. (2.1), application of Eq. (2.21) yields the radial distribution function $g(r)$.

The compressibility factor resulting from the virial EOS (2.4) is determined by the standard RFA method through the relations (5.10) and (D7). The result is

$$
Z_{v}(\eta)=1+2^{d-1} \eta a_{n+1}\left[1+\lambda \eta \sum_{j=0}^{n+1} \frac{(-1)^{d-j}}{(d-j) !} a_{j}\right]^{-1} .
$$

The isothermal susceptibility $\chi$ given by (4.13) can also be easily evaluated. On the one hand, from Eq. (4.14) and the definition of the $\gamma_{j}$ factors [Eq. [5.21)] one has

$$
H_{d-1}=(-1)^{n+1}[(d-2) ! !]^{2} \gamma_{d-2} .
$$

On the other hand, Eq. (5.20) at $l=d$ yields

$$
\gamma_{d-2}=-\left(D_{d}+\sum_{j=0}^{n} \gamma_{2 j} D_{d-2-2 j}\right) .
$$

Therefore, from Eq. (4.13) we obtain

$$
\chi(\eta)=1-2 \lambda(d-2) ! ! \eta\left(D_{d}+\sum_{j=0}^{n} \gamma_{2 j} D_{d-2-2 j}\right),
$$


with factors $\gamma_{0}, \gamma_{2}, \ldots, \gamma_{d-3}$ given by (5.25). Once $\chi$ is known, the thermodynamic relation (2.8) can be integrated to obtain $Z$ in the so-called compressibility route,

$$
Z_{c}(\eta)=\int_{0}^{1} \mathrm{~d} x \chi^{-1}(\eta x) .
$$

Additionally, one finds from (5.27) the following limit values

$$
Z_{v}(1)=\infty, \quad \chi(1)=0 .
$$

Comparison between the results obtained from Eqs. (5.28) and (5.32), which gives a measure of the degree of thermodynamic inconsistency in the standard RFA solution, will be presented in Sec. VII.

\section{Extended approximation}

It is possible to construct RFA solutions more elaborate than the standard RFA one by considering in Eq. (5.8) a number of unknowns $N+M+1$ larger than the number $3 n+4$ of basic constraints (5.7). We will refer to this case as the extended RFA. The simplest extension corresponds to

$$
N=d+1, \quad M=n+2,
$$

since Eq. (5.9) must be preserved. This involves two new parameters $\left(a_{n+2}\right.$ and $\left.b_{d+1}\right)$ which can be freely chosen without compromising the basic physical requirements. A natural choice is to adjust $a_{n+2}$ and $b_{d+1}$ by requiring prescribed values of the contact value of the radial distribution function, $g_{c} \equiv g\left(1^{+}\right)$, and of the isothermal susceptibility $\chi$. In practice, one can use (2.8) to evaluate $\chi$ from $g_{c}$ (or vice versa), so that only one EOS is needed and the thermodynamic consistency between the virial and the compressibility routes is thus guaranteed by construction.

With the choice (5.34), the relationship (5.10) can be used to eliminate one of the two new Padé coefficients (for instance, $\left.b_{d+1}\right)$ in terms of the other one $\left(a_{n+2}\right)$. Making use again of Eq. (D7), the extended RFA for $G(s)$ reads

$$
G(s)=\frac{e^{-s}}{s^{2}} \frac{A_{n+2}(s)}{1+g_{c}^{-1} a_{n+2} s+\lambda \eta \sum_{j=0}^{n+2} a_{j} \phi_{d-j}(s)} .
$$

The set of Padé coefficients $\left\{a_{j}, 0 \leq j \leq n+2\right\}$, is obtained in a manner similar to that given above. Thus, $a_{0}$ remains fixed by (5.14), while $a_{1}, \ldots, a_{n+1}$ are related to $a_{n+2}$ by the $n+1$ equations (5.26). So far, by setting $a_{n+2}=0$ we recover the standard RFA. However, now we fix the prescribed $\chi$ and then Eq. (5.31) provides the needed equation to close the set (5.26).

The nonlinearity of the problem in the extended RFA is higher than in the standard case, the number of mathematical solutions being $2^{n+1}$. In particular, one has to deal with a quadratic equation for $d=3[52,53,70]$, a quartic equation for $d=5$, and so on. It can be verified that in the zero-density limit the physical solution has the form

$$
\begin{array}{r}
\left.a_{j}\right|_{\eta=0}=\omega_{n+1, j}+\left.a_{n+2}\right|_{\eta=0} \omega_{n+1, j-1}, \\
\left.b_{j}\right|_{\eta=0}=\delta_{j, d}+\left.a_{n+2}\right|_{\eta=0} \delta_{j, d+1},
\end{array}
$$

where the numerical value of $\left.a_{n+2}\right|_{\eta=0}$ depends on the value of the fourth virial coefficient predicted by the prescribed EOS. Equations (5.36) and (5.37) imply that

$$
\begin{gathered}
\lim _{\eta \rightarrow 0} A_{n+2}(s)=\theta_{n+1}(s)\left(1+\left.a_{n+2}\right|_{\eta=0} s\right), \\
\lim _{\eta \rightarrow 0} B_{d+1}(s)=s^{d}\left(1+\left.a_{n+2}\right|_{\eta=0} s\right),
\end{gathered}
$$

so that Eq. (5.2) is recovered, irrespective of the chosen EOS (provided, of course, it is consistent with the exact second and third virial coefficients).

It is important to note that the physical root of the set of equations (5.26) and (5.31) must correspond to $a_{n+2}>0$. Otherwise, since $a_{0}=(d-2) ! !>0$, there would exist at least one positive real root $s_{0}$ of the polynomial $A_{n+2}(s)$. According to Eq. (5.35), this would imply $G\left(s_{0}\right)=0$, what is incompatible with a positive definite $g(r)$. A careful analysis of Eqs. (5.26) and (5.31) shows that the condition $a_{n+2}>0$ requires that the chosen values of $Z$ and $\chi$ satisfy the inequalities $Z>Z_{v}^{\mathrm{PY}}$ and $\chi>\chi_{c}^{\mathrm{PY}}$, where $Z_{v}^{\mathrm{PY}}$ and $\chi_{c}^{\mathrm{PY}}$ are the compressibility factor and the isothermal susceptibility, respectively, obtained from the standard RFA or, equivalently, from the PY solution. Therefore, if the prescribed $\chi$ is obtained from the prescribed $Z$ by application of Eq. (2.8), the extended RFA provides physical correlation functions only if

$$
Z_{v}^{\mathrm{PY}}<Z<Z_{c}^{\mathrm{PY}}
$$

When this condition is verified, the physical root of the set of equations (5.26) and (5.31) corresponds to the smallest positive real value of $a_{n+2}$.

\section{DIRECT CORRELATION FUNCTION}

According to Eq. (A12), the direct correlation function $c(r)$ can be evaluated from its Fourier transform as

$$
c(r)=\frac{(2 \pi)^{-(d+1) / 2}}{r^{d-2}} i \int_{-\infty}^{\infty} \mathrm{d} k k \widehat{c}(k) \theta_{n}(i k r) e^{-i k r} .
$$

In the RFA approach, using Eqs. (4.3), (5.4), and (5.8), we obtain

$$
S(k)=\frac{B^{+} B^{-}-A^{+} A^{-}}{\left(B^{+} e^{i k}-A^{+}\right)\left(B^{-} e^{-i k}-A^{-}\right)},
$$


where $A^{ \pm} \equiv \lambda \eta A_{M}( \pm i k)$ and $B^{ \pm} \equiv B_{N}( \pm i k)$. Next, use of Eq. (2.3) yields

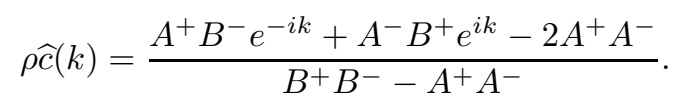

Since, according to Eqs. (4.7) and (5.4), one has $B_{N}(s) e^{s}-\lambda \eta A_{M}(s)=s^{d}[1+O(s)]$, it follows that the denominator in Eq. (6.2) is of order $k^{2 d}$. Therefore, the numerator must also be of order $k^{2 d}$ to have a finite value of $S(0)$. More specifically, for both the standard $(N=d$, $M=n+1)$ and the extended $(N=d+1, M=n+2)$ RFA approaches, one has

$$
B^{+} B^{-}-A^{+} A^{-}=\left(b_{d-1} b_{d+1}-b_{d}^{2}\right)(i k)^{2 d}+b_{d+1}^{2}(i k)^{2 d+2} .
$$

\section{A. Standard RFA}

In the case of the standard RFA, one has $b_{d+1}=0$, so that $B^{+} B^{-}-A^{+} A^{-}=-b_{d}^{2}(i k)^{2 d}$. Consequently, when inserting Eq. (6.3) into Eq. (6.1), each one of the three integrands has a pole of order $2 d-1$ at $k=0$. A residue analysis similar to that employed in Sec. III leads to

$$
c(r)=\frac{(-1)^{(d+1) / 2}}{r^{d-2}} \bar{R}_{4 n+4}(r) \Theta(1-r),
$$

where $\bar{R}_{4 n+4}(r)$ is a polynomial of degree $4 n+4=2 d-2$ given by

$$
\bar{R}_{4 n+4}(r)=\operatorname{Res}_{s=0}\left[\frac{A_{n+1}(-s) B_{d}(s)}{b_{d}^{2} s^{2 d-1}} \theta_{n}(s r) e^{s(1-r)}\right] .
$$

Comparison with Eq. (3.5) shows that, in the standard RFA, $c(r)$ has in the region $0 \leq r<1$ a polynomial form similar to that of $y_{1}(r)$ in the region $0 \leq r \leq 2$. As happened in the latter case, finiteness of $c(0)$ implies that $c(r)$ is a polynomial of degree $4 n+4-(d-2)=d$.

Equation (6.5) shows that $c(r)=0$ for $r>1$ in the standard RFA. Of course, the standard RFA complies with the physical requirement $g(r)=0$ for $r<1$. These two conditions define precisely the PY closure to solve the OZ equation for hard hyperspheres. Therefore, we find that the standard RFA, i.e., the approximation given by Eqs. (5.4) and (5.8) with the least number of coefficients satisfying the requirements (4.5) and (4.11), coincides with the PY solution for hard hyperspheres of odd dimensions [8, 11]. This is a remarkable result since both approaches are in principle rather independent. In fact, following the philosophy behind the RFA approach, it is straightforward to proceed to the first natural extension of the PY solution or standard RFA.

\section{B. Extended RFA}

In the extended RFA approach, $b_{d+1}=g_{c}^{-1} a_{n+2} \neq$ 0. As a consequence, Eq. (6.4) shows that, besides the pole of order $2 d-1$ at $k=0$, each one of the three contributions to $k \widehat{c}(k)$ has also two simple poles $k= \pm i \kappa$, $\kappa$ being a real quantity given by

$$
\kappa \equiv \sqrt{\left(b_{d}^{2}-b_{d-1} b_{d+1}\right) b_{d+1}^{-2}} .
$$

Therefore, applying the residue theorem we get

$$
c(r)=(-1)^{(d+1) / 2}\left(K_{+}+K_{-}-K_{0}\right) \frac{\theta_{n}(\kappa r) e^{-\kappa r}}{r^{d-2}}
$$

for $r>1$ and

$$
\begin{aligned}
c(r)= & -\frac{(-1)^{(d+1) / 2}}{r^{d-2}}\left[\widetilde{R}_{4 n+4}(r)+K_{-} \theta_{n}(-\kappa r) e^{\kappa r}\right. \\
& \left.+\left(K_{0}-K_{-}\right) \theta_{n}(\kappa r) e^{-\kappa r}\right]
\end{aligned}
$$

for $r<1$. In the above expressions,

$$
\begin{gathered}
K_{ \pm} \equiv \frac{A_{n+2}(\mp \kappa) B_{d+1}( \pm \kappa) e^{ \pm \kappa}}{2 b_{d+1}^{2} \kappa^{2 d}} \\
K_{0} \equiv \lambda \eta \frac{A_{n+2}(\kappa) A_{n+2}(-\kappa)}{b_{d+1}^{2} \kappa^{2 d}} \\
\widetilde{R}_{4 n+4}(r) \equiv \operatorname{Res}_{s=0}\left[\frac{A_{n+2}(-s) B_{d+1}(s)}{b_{d+1}^{2} s^{2 d-1}\left(s^{2}-\kappa^{2}\right)} \theta_{n}(s r) e^{s(1-r)}\right]
\end{gathered}
$$

Equation (6.8) shows that, in contrast to the PY solution (or, equivalently, the standard RFA), $c(r)$ does not vanish outside the core in the extended RFA. In fact, $c(r) \propto \theta_{n}(\kappa r) e^{-\kappa r} / r^{d-2}$ for $r>1$. This functional form can be considered as an extension to odd $d$ of the wellknown Yukawa form $e^{-\kappa r} / r$ for $d=3$ [72.

The form (6.8) obtained from the extended RFA for hard hyperspheres admits an alternative interpretation. Imagine an interaction potential with a hard core at $r=1$ plus an attractive Yukawa tail $-\epsilon \theta_{n}(z r) e^{-z r} / r^{d-2}$ for $r>1$. In the mean spherical approximation (MSA) 1] the closure to the $\mathrm{OZ}$ relation would be $c(r)=$ $\left(\epsilon / k_{B} T\right) \theta_{n}(z r) e^{-z r} / r^{d-2}$ for $r>1$, which has the same form as Eq. (6.8). As a consequence, the MSA for the hard-core Yukawa interaction with $d=$ odd is exactly solvable, the corresponding functions $G(s), S(k)$, and $c(r)$ for $r<1$ being given by Eqs. (5.35), (6.2) (with $N=d+1$ and $M=n+2$ ), and (6.9), respectively. The main difference between the extended RFA for hard hyperspheres and the MSA for the hard-core Yukawa potential is that, while in the former case the two input parameters are the contact value $g_{c}=g\left(1^{+}\right)$and the isothermal susceptibility $\chi$, in the latter case the control parameters are the reduced temperature $k_{B} T / \epsilon$ and the inverse interaction range $z$.

In the three-dimensional case, the extended RFA [52] reproduces the the so-called generalized mean spherical approximation (GMSA) for hard spheres [73], which consists of closing the $\mathrm{OZ}$ equation with the assumption that $c(r)$ has a Yukawa tail outside the core $(r>1)$. From that point of view, the extended RFA applied to higher odd dimensions can be seen as the natural extension of the GMSA to those dimensions. 


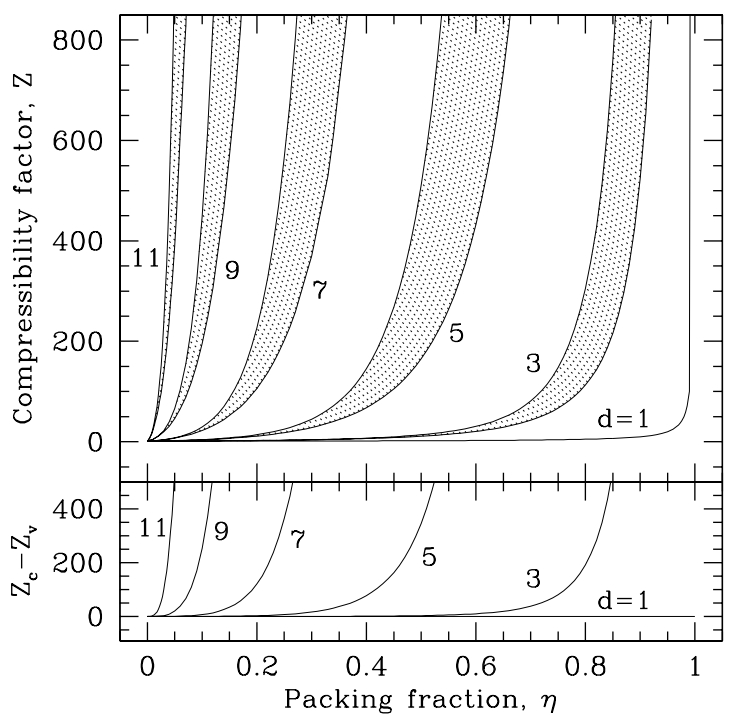

FIG. 2: Top panel: Compressibility factors $Z_{v}^{\mathrm{PY}}$ and $Z_{c}^{\mathrm{PY}}$ (curves on the right and left edges of each shaded area, respectively) arising from the virial and the compressibility routes according to the standard RFA method (PY theory) at dimensions $d=1,3,5,7,9$, and 11, as functions of the packing fraction $\eta$. Bottom panel: Difference between $Z_{c}^{\mathrm{PY}}$ and $Z_{v}^{\mathrm{PY}}$.

TABLE III: Packing fractions at freezing transition $\left(\eta_{f}\right)$ and closest packing $\left(\eta_{c}\right)$ predicted for fluids at $d=1,3,5,7,9,11$.

\begin{tabular}{ccc}
\hline \hline$d$ & $\eta_{f}$ & $\eta_{c}$ \\
\hline 1 & - & 1 \\
3 & $0.494^{a}$ & $\sqrt{2} \pi / 6 \approx 0.74048^{e}$ \\
5 & $0.19^{b}$ & $\sqrt{2} \pi^{2} / 30 \approx 0.46526^{e}$ \\
7 & $0.072^{c}$ & $\pi^{3} / 105 \approx 0.29530^{e}$ \\
9 & $0.027^{d}$ & $\sqrt{2} \pi^{4} / 945 \approx 0.14578^{e}$ \\
11 & $0.009^{d}$ & $32 \pi^{5} /(93555 \sqrt{3}) \approx 0.06043^{e}$ \\
\hline \hline
\end{tabular}

${ }^{a}$ Monte Carlo simulations [5]

${ }^{b}$ Molecular dynamics simulations 6

${ }^{c}$ Molecular dynamics simulations 31 ]

${ }^{d}$ Estimated by the method of Refs. [24, 30]

${ }^{e}$ Densest lattice packing listed in Ref. [74].

\section{RESULTS}

\section{A. Standard RFA (PY theory)}

We list in Appendix E the explicit solutions of Eq. (5.26) corresponding to the standard RFA approach (or, equivalently, the PY theory) for fluids in dimensions $d=$ $1,3,5$. The solution for $d=7$ is also analytical and agrees with the results reported in Refs. 31, [43]. The solutions to Eq. (5.26) for $d=9,11$ have been obtained numerically.

The compressibility factors $Z_{v}^{\mathrm{PY}}$ and $Z_{c}^{\mathrm{PY}}$ derived by the standard RFA approach from the virial and compressibility routes [Eqs. (5.28) and (5.32)], respectively,

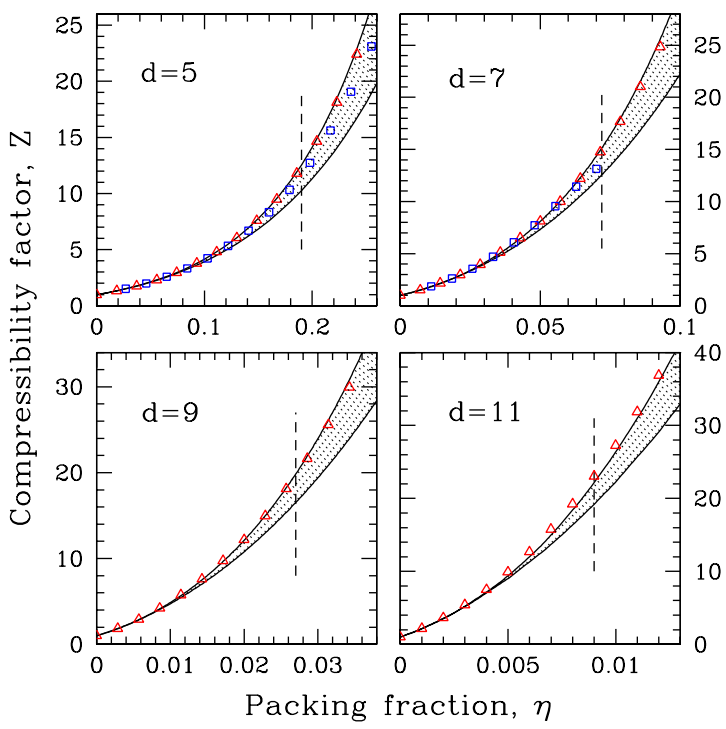

FIG. 3: (Color online) Compressibility factors $Z_{c}$ and $Z_{v}$ arising from the standard RFA (edges of shaded areas) for $d=5,7,9,11$. The values obtained from the SMS EOS [17] for $d=5,7,9,11$ (triangles), and the LM EOS [19] for $d=5,7$ (squares) are also shown. The vertical dashed lines mark the freezing packing fraction reported in Table III

are shown in Fig. 2 for $d=1,3,5,7,9,11$, as functions of the packing fraction. Both routes yield identical and exact results only in the case $d=1$. Discrepancies between $Z_{c}^{\mathrm{PY}}$ and $Z_{v}^{\mathrm{PY}}$ grow noticeably with increasing dimension for $d \geq 3$ (bottom panel in Fig. 22). The compressibility factors predicted by the standard RFA have a singularity at $\eta=1$ for all $d$ [cf. Eq. [5.33)]. However, since $d$-spheres are not space filling (except for $d=1$ ) the true pressure must present a singularity at a certain density lower than or equal to the closest packing fraction $\eta_{c}$. The maximal packing fractions presently known in these dimensions are listed in the third column of Table III. In practice, the inconsistency between $Z_{v}^{\mathrm{PY}}$ and $Z_{c}^{\mathrm{PY}}$ is not as severe as it is appears in Fig. 2 because the fluid phase is actually limited to very low values of $\eta$ for high dimensions. Freezing transitions are predicted for hard $d$-sphere fluids with $d>1$ and found to occur at increasing lower packing fractions with increasing dimensionality [6, 30, 41]. Some values of the freezing packing fraction $\eta_{f}$ are listed in the second column of Table [III Figure 3 depicts the compressibility factors $Z_{c}^{\mathrm{PY}}$ and $Z_{v}^{\mathrm{PY}}$ up to densities in the neighborhood of the liquid-solid phase transition for fluids with $d=5,7,9,11$. The figure also includes the values of $Z$ predicted by the extension of the Carnahan-Starling EOS proposed by Song, Mason, and Stratt (SMS) [17], as well as the predictions of the semi-empirical EOS proposed by Luban and Michels (LM) 19]. There is a reasonable agreement among these two latter EOS and the PY results via the compressibility route within the fluid phase. In fact, comparison of $Z_{v}^{\mathrm{PY}}$ and $Z_{c}^{\mathrm{PY}}$ with com- 


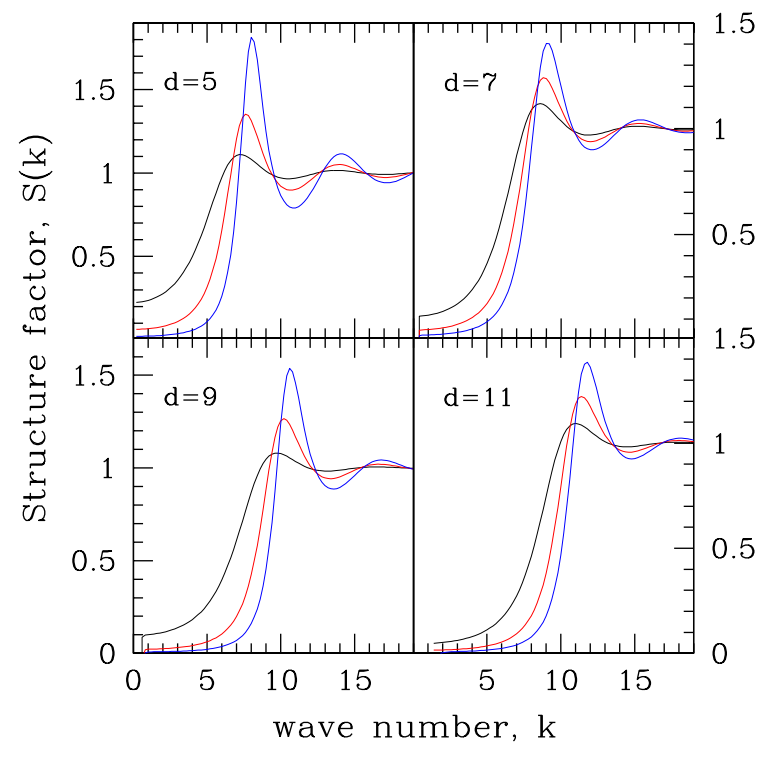

FIG. 4: (Color online) The structure factor as obtained from the standard RFA (or PY solution) for hyperspheres in $d=5$ (at $\eta=0.06,0.13,0.2), d=7$ (at $\eta=0.03,0.05,0.07$ ), $d=9$ (at $\eta=0.01,0.025,0.04)$, and $d=11$ (at $\eta=$ $0.005,0.01,0.015)$. The first peak increases with increasing density.

puter simulations in the cases $d=5$ [28] and $d=7$ [31] shows that, in the stable fluid region, the true values of $Z$ are bracketed by $Z_{v}^{\mathrm{PY}}$ and $Z_{c}^{\mathrm{PY}}$ in the form indicated by Eq. (5.40), being closer to $Z_{c}^{\mathrm{PY}}$ than to $Z_{v}^{\mathrm{PY}}$. This in passing gives further support to the extended RFA, which requires as input an EOS satisfying Eq. (5.40) in order to provide physically meaningful results.

Figure 4 shows the structure factor obtained from the standard RFA method for fluids in dimensions $d=$ $5,7,9,11$ for densities near the values of $\eta_{f}$ given in Table [II]. As expected, the oscillations of $S(k)$ become more pronounced as the density increases. We can also observe that the location of the first peak tends to move to higher wavenumbers as the dimensionality increases [41].

\section{B. Extended RFA}

As seen in Sec.VI, the main signature of the extended RFA solution with respect to the standard one (or PY solution) is that the former yields a direct correlation function with a (generalized) Yukawa form outside the core [cf. Eq. (6.8)]. In Fig. 5 we compare $c(r)$ evaluated from the standard and extended RFA (the latter being complemented by the SMS and LM EOS) for $d=5$ and $d=7$ at densities close to the respective values of $\eta_{f}$. At those densities, as shown in Fig. 3. the SMS EOS is closer to the PY compressibility route than the LM EOS. As a consequence, the value of the extended coefficient $a_{n+2}$ is smaller in the former case than in the

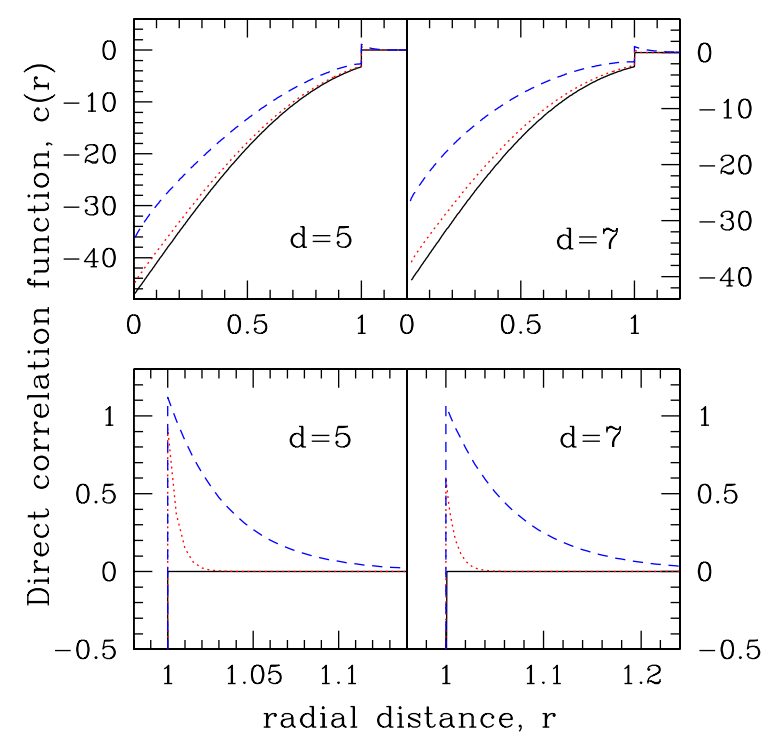

FIG. 5: (Color online) Top panels: Direct correlation function for $d=5$ (at $\eta=0.2$ ) and $d=7$ (at $\eta=0.07$ ) as computed from the standard RFA (solid lines) and the extended RFA using the SMS EOS [17] (dotted lines) and the LM EOS [19] (dashed lines). Bottom panels: Details of the Yukawa tails outside the core as obtained from the extended RFA solutions.

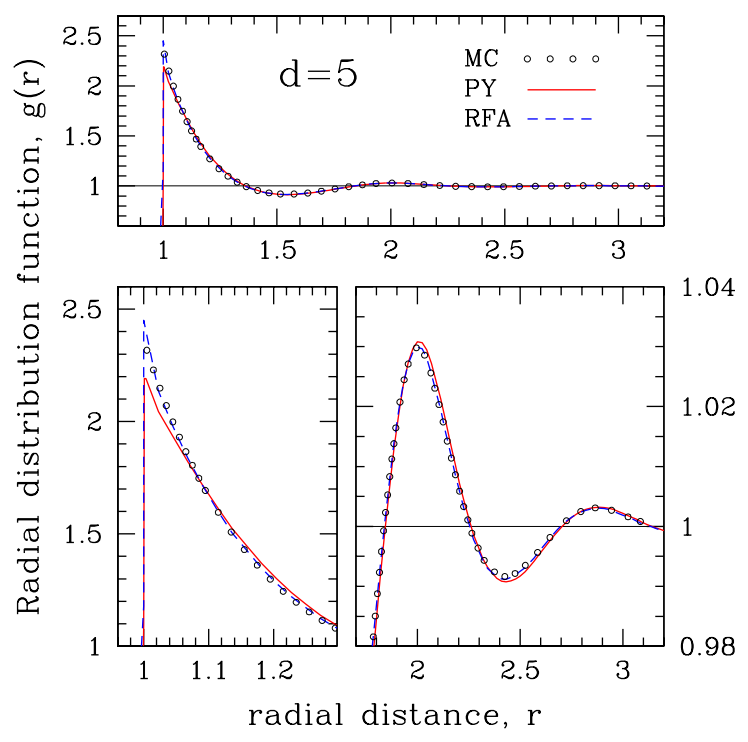

FIG. 6: (Color online) Radial distribution function of hard hyperspheres in $d=5$ obtained from the extended RFA method (dashed line), the PY solution (solid line), and Monte Carlo simulations [37] (symbols) at $\rho=0.8(\eta \approx 0.1316)$. The top panel shows the global behavior, while the bottom panels show the details near the first peak (left) and between the second and third peaks (right). 


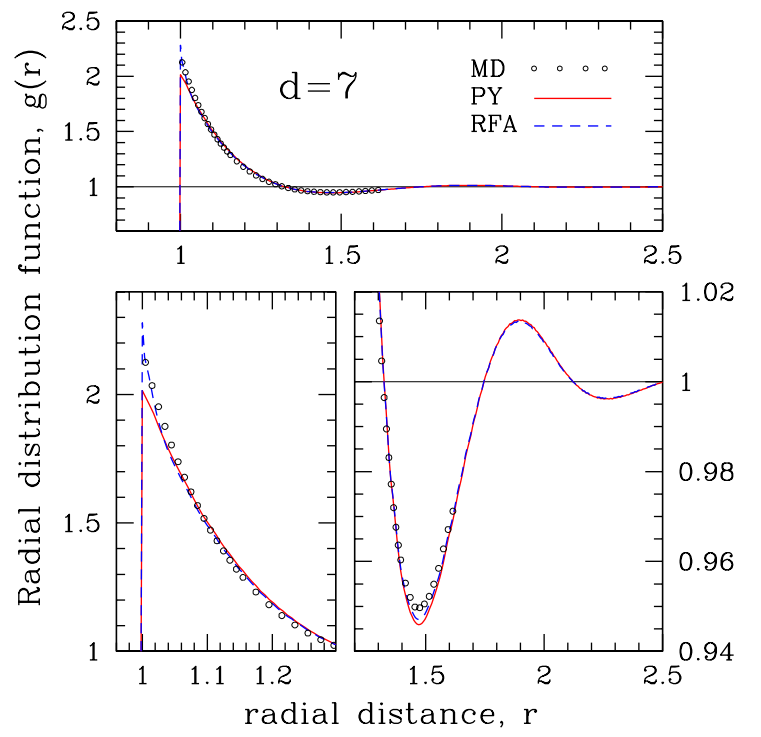

FIG. 7: (Color online) Radial distribution function of hard hyperspheres in $d=7$ obtained from the extended RFA method (dashed line), the PY solution (solid line), and molecular dynamics simulations [40, 45] (symbols), at $\rho=1.4$ $(\eta \approx 0.05168)$. The top panel shows the global behavior, while the bottom panels show the details near the first peak (left) and near the first minimum (right).

latter. This explains the fact that in Fig. 5 the curves corresponding to the extended RFA complemented by the SMS EOS are much closer to the PY ones (and with a weaker Yukawa tail) than those obtained by using the LM EOS. Given the semi-empirical character of the LM EOS and its excellent agreement with computer simulations [19, 31], it is reasonable to expect that the true $c(r)$ is better represented by the extended RFA complemented with the LM EOS than with the SMS EOS. Of course, there are other EOS proposed in the literature for hard hyperspheres [16, 18, 20, 25], but the ones considered in Fig. 5 are enough for illustrative purposes.

Comparisons between the pair distribution function obtained from both RFA approaches and available computer simulations [37, 40, 45] are shown in Figs. 66 and 7 for $d=5$ and $d=7$, respectively. The extended RFA results have been obtained with the SMS EOS, but we have checked that no significant differences are found if the LM EOS is used instead. This indicates that $g(r)$ is much less sensitive than $c(r)$ to the choice of the input EOS. Figures 6 and 7 show that the standard RFA (or PY solution) provides an accurate estimate of $g(r)$ for all radial distances at the considered densities $\left(\eta \approx 0.7 \eta_{f}\right)$, although some small discrepancies appear near the first maxima and minima, especially at contact. These deviations are substantially corrected by the extended RFA.

\section{CONCLUDING REMARKS}

In this work we have generalized the RFA method, originally developed for three-dimensional hard-sphere fluids [52, 53, 70], to hypersphere systems in arbitrary odd dimensions $d$, providing explicit results for $d \leq 11$. This generalization is not trivial at all. In the application of the RFA approach, one must define a functional $G(s)$ of $g(r)$ in Laplace space, introduce an auxiliary function $\Psi(s)$ directly related to $G(s)$, and approximate $\Psi(s)$ by a rational function (or Padé approximant), determining the coefficients by the application of basic consistency conditions.

In the one- and three-dimensional cases the key function $G(s)$ is the Laplace transform of $g(r)$ and $r g(r)$, respectively [53, 55]. Thus one might be tempted to define $G(s)$ for $d=$ odd simply as the Laplace transform of $r^{(d-1) / 2} g(r)$. Here, however, we have adopted the criterion that $G(s)$ must be defined as to be closely related to the static structure factor $S(k)$ of the fluid. This has led us to the definition (4.1) and to the relationship (4.3), where the reverse Bessel polynomials (2.24) play a central role. As a byproduct, we have derived a general polynomial expression for the overlap volume of two identical hyperspheres, this quantity providing the cavity function to first order in density, $y_{1}(r)$. Once $G(s)$ has been identified, one needs to define the auxiliary function $\Psi(s)$ to be approximated by a rational function. Using the exact knowledge of $G(s)$ to first order in density, Eqs. (5.1)(5.3), it turns out that the natural definition of $\Psi(s)$ is provided by Eq. (5.4). Finally, $\Psi(s)$ has been approximated by a rational function, Eq. (5.8), the degree difference between the numerator and denominator being fixed by the exact large- $s$ behavior of $G(s)$. The coefficients in the Padé approximant for $\Psi(s)$ are constrained to fit the exact small-s behavior of $G(s)$ or, equivalently, the small- $k$ behavior of $S(k)$. This representation reproduces the exact expansion in density of $S(k)$ up to second order.

We have called standard RFA to the case in which the number of parameters in the Padé approximant equals the number of constraints. It turns out that the associated direct correlation function vanishes outside the core. Therefore, quite interestingly, the standard RFA coincides with the solution of the PY closure to the OZ relation for hard-particle fluids in all odd dimensions [8, 11] . This equivalence between two completely independent paths allows one to view the PY solution for hard hyperspheres as the simplest one of a broad class of approximations. In fact, a more flexible approximation is obtained by adding a pair of new terms (one in the numerator and the other one in the denominator) in the Padé approximant for $\Psi(s)$, resulting in what we have called the extended RFA. Apart from satisfying the small- $k$ behavior of $S(k)$, the parameters are determined by requiring thermodynamic consistency with a prescribed EOS, which must satisfy the inequalities (5.40) to ensure that the extended RFA solution is positive definite. 
Comparison with available computer simulations for $d=5$ [37] and $d=7$ [40, 45] shows that the radial distribution function predicted by the standard RFA (or PY solution) is rather accurate. On the other hand, there exist certain small discrepancies (especially near contact) that are satisfactorily corrected by the extended RFA.

The work presented in this paper is aimed at contributing to our understanding of the structural properties of hard-hypersphere fluids and to the mathematical intricacies of their statistical-mechanical description. Moreover, this work paves the path to the study of other related systems in $d$ dimensions, such as sticky hard hyperspheres, square-well particles, or multicomponent hardhypersphere fluids. Work is now in progress along these lines and the results will be published elsewhere.

\section{Acknowledgments}

We thank Santos Bravo Yuste, Mariano López de Haro, and Salvatore Torquato for helpful comments. We are especially grateful to Marvin Bishop for his suggestions and for providing us with tables of the computer simulation data of Refs. [37], [40], and [45]. One of the authors (R.D.R.) acknowledges the Carrera del Investigador Científico, Consejo de Investigaciones Científicas y Técnicas de la Nación (CONICET, Argentina). This work has been supported by the SeCyT-UNC (Argentina) through Grant No. 162/06, by the Ministerio de Educación y Ciencia (Spain) through Grant No. FIS2007-60977 (partially financed by FEDER funds), and by the Junta de Extremadura (Spain) through Grant No. GRU07046.

\section{APPENDIX A: FOURIER TRANSFORM USING REVERSE BESSEL POLYNOMIALS}

If the function $\xi(\mathbf{r})=\xi(r)$ is isotropic, Eqs. (2.18) and (2.19) become [75]

$$
\begin{aligned}
& \widehat{\xi}(k)=(2 \pi)^{d / 2} \int_{0}^{\infty} \mathrm{d} r r^{d-1} \xi(r) \frac{J_{d / 2-1}(k r)}{(k r)^{d / 2-1}}, \\
& \xi(r)=\frac{1}{(2 \pi)^{d / 2}} \int_{0}^{\infty} \mathrm{d} k k^{d-1} \widehat{\xi}(k) \frac{J_{d / 2-1}(k r)}{(k r)^{d / 2-1}},
\end{aligned}
$$

respectively. Here $k=|\mathbf{k}|$ is the magnitude of the wave vector and $J_{\nu}(x)$ is the Bessel function of the first kind of order $\nu$. For half-integer order $\nu=n+1 / 2$, it is usual to introduce the spherical Bessel function of the first kind $j_{n}(x)$ given by

$$
j_{n}(x)=\sqrt{\frac{\pi}{2 x}} J_{n+1 / 2}(x) .
$$

In order to rewrite the Fourier transform in a more appropriate form, we express the spherical Bessel functions as follows:

$$
\begin{aligned}
j_{n}(x) & =\frac{\theta_{n}(-i x) e^{i x}-\theta_{n}(i x) e^{-i x}}{2 i x^{n+1}} \\
& =\frac{1}{x^{n+1}} \operatorname{Im}\left[\theta_{n}(-i x) e^{i x}\right],
\end{aligned}
$$

where the reverse Bessel polynomial $\theta_{n}(t)$ is defined by Eq. (2.24) 76.

A useful integral identity for $\theta_{n}(t)$, which we have not found in the literature, is

$$
\theta_{n}(t) e^{-t}=\int_{t}^{\infty} \mathrm{d} z z \theta_{n-1}(z) e^{-z}
$$

By taking the derivative of both sides of Eq. A5 one obtains

$$
\frac{\mathrm{d}}{\mathrm{d} t}\left[\theta_{n}(t) e^{-t}\right]=-t \theta_{n-1}(t) e^{-t} .
$$

Next, using Eq. (A4), it is easy to prove that the recurrence relation of the spherical Bessel functions [77],

$$
\frac{\mathrm{d}}{\mathrm{d} x} j_{n}(x)=-\frac{n+1}{x} j_{n}(x)+j_{n-1}(x),
$$

is recovered. In passing, from Eq. (A6) we may note the recurrence formula

$$
\frac{\mathrm{d}}{\mathrm{d} t} \theta_{n}(t)=\theta_{n}(t)-t \theta_{n-1}(t) .
$$

Another recurrence relation is 64]

$$
\theta_{n}(t)=(2 n-1) \theta_{n-1}(t)+t^{2} \theta_{n-2}(t) .
$$

Besides, with the help of Eq. (A4) and expression (10.1.2) of Ref. 77], one can find the asymptotic relation

$$
\frac{i}{2}\left[\theta_{n}(i x) e^{-i x}-\theta_{n}(-i x) e^{i x}\right]=\frac{x^{2 n+1}}{(2 n+1) ! !}\left[1+O\left(x^{2}\right)\right],
$$

which is used in the evaluation of $y_{1}(r)$ in Sec. III

We return now to the problem of expressing Fourier transforms in odd dimensions. The functions $\xi(r)$ and $\widehat{\xi}(k)$ can be extended to negative $r$ and $k$ as $\xi(-r)=\xi(r)$ and $\widehat{\xi}(-k)=\widehat{\xi}(k)$, respectively. Then, with the help of (A3) and (A4) we can rewrite (A1) and (A2) as

$$
\begin{gathered}
\widehat{\xi}(k)=\frac{(2 \pi)^{(d-1) / 2}}{k^{d-2}} i \int_{-\infty}^{\infty} \mathrm{d} r r \xi(r) \theta_{n}(i k r) e^{-i k r}, \\
\xi(r)=\frac{(2 \pi)^{-(d+1) / 2}}{r^{d-2}} i \int_{-\infty}^{\infty} \mathrm{d} k k \widehat{\xi}(k) \theta_{n}(i k r) e^{-i k r},
\end{gathered}
$$

respectively, where $n$ is defined by Eq. (2.22). Finally, introducing the functional (2.23), one arrives at Eqs. (2.20) and (2.21).

It is worthwhile noting that if $\theta_{n}(x)$ is replaced by its polynomial expression (2.24), then $\mathcal{F}_{n}[\xi(x)]$ can be 
expressed in terms of the Laplace transforms of $x^{j+1} \xi(x)$ with $0 \leq j \leq n$,

$$
\begin{gathered}
\mathcal{F}_{n}[\xi(x)](s)=\sum_{j=0}^{n} \omega_{n, j} s^{j} \mathcal{L}\left[x^{j+1} \xi(x)\right](s), \\
\mathcal{L}[\xi(x)](s) \equiv \int_{0}^{\infty} \mathrm{d} x \xi(x) e^{-s x} .
\end{gathered}
$$

\section{APPENDIX B: EVALUATION OF $P_{n+1}(r)$}

In this Appendix we obtain an explicit expression for the polynomial $P_{n+1}(r)$ related by Eq. (3.9) to the firstorder cavity function $y_{1}(r)$. The derivation of $P_{n+1}(r)$ from relations (3.7) and (3.8) is straightforward but rather tedious. We found it more practical to use a known expression for the scaled overlap volume in terms of the normalized incomplete beta function [16],

$$
\Omega_{d}(r) \equiv \frac{y_{1}(r)}{y_{1}(0)}=\frac{B_{1-r^{2} / 4}((d+1) / 2,1 / 2)}{B((d+1) / 2,1 / 2)} \Theta(2-r),
$$

where

$$
B_{x}(a, b)=\int_{0}^{x} \mathrm{~d} t t^{a-1}(1-t)^{b-1}
$$

is the incomplete beta function and $B(a, b)=$ $B_{x=1}(a, b)=\Gamma(a) \Gamma(b) / \Gamma(a+b)$ is the beta function. In the present case, for $d$ odd,

$$
B((d+1) / 2,1 / 2)=\frac{2^{(d+1) / 2}((d-1) / 2) !}{d ! !} .
$$

From (B1)-(B3), it is straightforward to obtain

$$
\frac{\mathrm{d} \Omega_{d}(r)}{\mathrm{d} r}=-\frac{d ! !}{2^{(d+1) / 2}((d-1) / 2) !}\left(1-\frac{r^{2}}{4}\right)^{(d-1) / 2} \Theta(2-r) .
$$

Expanding the binomial and integrating over $r$, one has

$$
\Omega_{d}(r)=1-\frac{(2 n+3) ! ! r}{2^{n+2}} \sum_{j=0}^{n+1} \frac{\left(-r^{2} / 4\right)^{j}}{(2 j+1) j !(n+1-j) !},
$$

where it has been implicitly assumed that $r \leq 2$.

On the other hand, inserting Eq. (3.9) into the definition of $\Omega_{d}(r)$ we get

$$
\Omega_{d}(r)=\frac{(2 n+3) ! !}{2}(2-r)^{n+2} P_{n+1}(r),
$$

where we have taken into account that, since $y_{1}(0)=$ $2^{d} v_{d}$, then $P_{n+1}(0)=2^{-(n+1)} /(2 n+3)$ !!. From Eqs. (B5) and (B6) one finally gets

$$
P_{n+1}(r)=\sum_{j=0}^{n+1} p_{n+1, j} r^{j}
$$

with the coefficients

$$
\begin{aligned}
p_{m, j}= & \frac{2^{-(m+j)}}{m !}\left[\frac{(m+j) !}{j !(2 m+1) ! !}\right. \\
& \left.-2^{-m} \sum_{l=0}^{[(j-1) / 2]} \frac{(-1)^{l}(m-1+j-2 l) !}{(2 l+1)(j-1-2 l) ! l !(m-l) !}\right],
\end{aligned}
$$

where $[(j-1) / 2]$ represents the integer part of $(j-1) / 2$.

Equation (B5) coincides with the expression (3-24) of Ref. [42] evaluated in the case $d=$ odd. The novel contribution of the procedure outlined in Sec. III is to show the factorization of the overlap volume [Eqs. (3.9) and (B6)] into the product of $(2-r)^{n+2}$ and $P_{n+1}(r)$, which is not evident from Eqs. (B1), (B4), or (B5).

\section{APPENDIX C: EVALUATION OF $G_{1}(s)$}

In this Appendix the function $G_{1}(s)$ defined by Eq. (4.17) is evaluated. We start from the identity

$$
\int \mathrm{d} r r^{m}(s r)^{j} e^{-s r}=-\frac{e^{-s r}}{s^{m+1}}(m+j) ! \sum_{l=0}^{m+j} \frac{(s r)^{l}}{l !} .
$$

Consequently,

$$
\begin{aligned}
\int \mathrm{d} r r^{m} \theta_{n}(s r) e^{-s r}= & -\frac{e^{-s r}}{s^{m+1}} \sum_{j=0}^{n}(m+j) ! \omega_{n, j} \\
& \times \sum_{l=0}^{m+j} \frac{(s r)^{l}}{l !}
\end{aligned}
$$

Finally, making use of Eq. (B6), we get

$$
\frac{2^{2 n+3}}{y_{1}(0)} \int \mathrm{d} r r y_{1}(r) \theta_{n}(s r) e^{-s r}=-\frac{e^{-s r}}{s^{2 n+5}} Q_{3 n+4}(r, s),
$$

where we have called

$$
\begin{aligned}
Q_{3 n+4}(r, s) \equiv & (2 s)^{2 n+3} \sum_{j=0}^{n}(j+1) ! \omega_{n, j} \sum_{l=0}^{j+1} \frac{(s r)^{l}}{l !} \\
& -\frac{(2 n+3) ! !}{2^{n+1}} \sum_{m=0}^{n+1} \frac{(-1)^{m}\left(4 s^{2}\right)^{n+1-m}}{(2 m+1) m !(n+1-m) !} \\
& \times \sum_{j=0}^{n}(2 m+2+j) ! \omega_{n, j} \sum_{l=0}^{2 m+2+j} \frac{(s r)^{l}}{l !}(\mathrm{C} 4)
\end{aligned}
$$

Equations (4.17) and (C3) allow us to write

$$
G_{1}(s)=s^{-(2 n+5)}\left[e^{-s} Q_{3 n+4}(1, s)-e^{-2 s} Q_{3 n+4}(2, s)\right] .
$$

The function $Q_{3 n+4}(2, s)$ can be further simplified. To that end, note that, according to Eq. (C3),

$$
\mathcal{F}_{n}\left[y_{1}(r)\right](s)=\frac{v_{d}}{s^{2 n+5}}\left[Q_{3 n+4}(0, s)-e^{-2 s} Q_{3 n+4}(2, s)\right] .
$$


Using Eq. (2.20) and comparing with Eq. (3.4) one obtains

$$
\begin{gathered}
Q_{3 n+4}(2, s)=(-1)^{n} 4^{n+1}(2 n+3) ! ! \theta_{n+1}^{2}(s), \quad(\mathrm{C} 7) \\
Q_{3 n+4}(0, s)+Q_{3 n+4}(0,-s)= \\
\begin{aligned}
& (-1)^{n} 2^{2 n+3}(2 n+3) ! ! \\
& \times \theta_{n+1}(s) \theta_{n+1}(-s) .
\end{aligned}
\end{gathered}
$$

Equation (4.18) is obtained by inserting Eq. (C7) into Eq. (C5), using Eqs. (4.16) and (4.19), and calling $Q_{3 n+4}(s) \equiv Q_{3 n+4}(1, s)$.

\section{APPENDIX D: CONSTRAINTS ON THE PADÉ COEFFICIENTS}

In this Appendix we consider the determination of the Padé coefficients $\left\{a_{j}\right\}$ and $\left\{b_{j}\right\}$ by application of the physical constraints (5.7) or, equivalently, (4.11). Substitution of Eq. (5.4) into Eq. (4.7), with the term $e^{s}$ expanded in power series, yields

$$
\sum_{j=0}^{M} \widetilde{a}_{j} s^{d+j}=\sum_{j=0}^{\infty} C_{j} s^{j}+\sum_{j=2}^{\infty}\left(\sum_{l=0}^{j-2} \gamma_{j-2-l} C_{l}\right) s^{j},
$$

where $C_{j}$ is the $j$ th coefficient in the series expansion of $B_{N}(s) e^{s}-\lambda \eta A_{M}(s)$, i.e.,

$$
\begin{array}{r}
C_{j}=\sum_{l=0}^{j} \frac{b_{l}}{(j-l) !}-\lambda \eta a_{j}, \quad 0 \leq j \leq M, \\
C_{j}=\sum_{l=0}^{\min (j, N)} \frac{b_{l}}{(l-k) !}, \quad j \geq M+1,
\end{array}
$$

and $\widetilde{a}_{j}$ and $\gamma_{j}$ are defined in Eq. (5.21). From the analysis of Eq. (D1) one obtains

$$
\begin{gathered}
C_{j}=0, \quad 0 \leq j \leq d-1, \\
C_{j}=\widetilde{a}_{j-d}, \quad d \leq j \leq d+1, \\
C_{d+m}+\sum_{j=0}^{m-2} \gamma_{m-2-j} C_{d+j}= \begin{cases}\widetilde{a}_{m}, & 2 \leq m \leq M \\
0, & m \geq M+1 .\end{cases}
\end{gathered}
$$

Taking into account that, as a consequence of Eq. (4.10), the first $n$ factors $\gamma_{j}$ with $j=$ odd vanish, it is obvious that (D4)-(D6) provide a total of $(3 d+1) / 2=3 n+5$ equations without unknown $\gamma_{j}$ factors. Therefore, in agreement with Eq. (5.7), this is the number of constraints on the Padé coefficients $\left\{b_{j}\right\}$ and $\left\{a_{j}\right\}$.
In particular, Eqs. (D4) and (D5), together with the definitions (D2) and (D3), allow one to express the coefficients $\left\{b_{j}\right\}$ in terms of $\left\{a_{j}\right\}$ for the cases $N=d$ and $N=d+1$,

$$
b_{j}=\delta_{j, d}+\lambda \eta \sum_{l=0}^{\min (j, M)} \frac{(-1)^{j-l}}{(j-l) !} a_{l}, \quad 0 \leq j \leq d,
$$

$$
b_{d+1}=\widetilde{a}_{1}-1+\lambda \eta \sum_{l=0}^{M} \frac{(-1)^{j-l}}{(j-l) !} a_{l},
$$

where in Eq. (D7) we have taken into account that $\widetilde{a}_{0}=$ 1. Notice that if $N=d$, then $b_{d+1}=0$ and Eq. (D8) provides an expression for one unknown $a_{j}$ in terms of the other ones. Expression (D7) can be verified by induction using (D4) and the following algebraic identity,

$$
\sum_{j=0}^{m-1} \frac{(-1)^{j}}{j !(m-j) !}=\frac{(-1)^{m-1}}{m !} .
$$

\section{APPENDIX E: SOLUTIONS OF THE STANDARD RFA FOR $d=1,3$, AND 5}

The explicit solutions of Eq. $(\underline{5.26)}$ are listed here for hard $d$-sphere systems in $d=1,3$, and 5 within the standard RFA approach presented in Sec. V

\section{Hard rods}

The one-dimensional case $(d=1)$ corresponds to $n=$ -1 . The recursive relation (A9) yields $\theta_{-1}(t)=1 / t$, so that, according to Eq. (4.1),

$$
G(s)=s^{-1} \int_{0}^{\infty} \mathrm{d} r g(r) e^{-s r} .
$$

Therefore, $s G(s)$ is the Laplace transform of $g(r)$. Taking $n=-1$ in Eq. (5.18) with $\lambda=1$ [cf. Eq. (4.19)], one simply gets

$$
s G(s)=\frac{1}{[\eta+(1-\eta) s] e^{s}-\eta} .
$$

The Laplace transform $s G(s)$ can be easily inverted to obtain the well-known radial distribution function for hard rods [48]. Thus, the standard RFA becomes exact for $d=1$.

\section{Hard spheres}

In the case $d=3$ or $n=0$, Eq. (4.1) becomes

$$
G(s)=\int_{0}^{\infty} \mathrm{d} r r g(r) e^{-s r},
$$


and so $G(s)$ represents the Laplace transform of $r g(r)$. According to Eq. (5.18),

$$
G(s)=\frac{e^{-s}}{s^{2}} \frac{1+a_{1} s}{1-12 \eta\left[\phi_{3}(s)+a_{1} \phi_{2}(s)\right]},
$$

where we have taken into account that $\lambda=-12$ and $a_{0}=1$. Equation (5.26) reduces to $D_{1}=\widetilde{a}_{1}=a_{1}$, whose solution is

$$
a_{1}=\frac{1+\eta / 2}{1+2 \eta} .
$$

From here one can easily get

$$
g\left(1^{+}\right)=\frac{1+\eta / 2}{(1-\eta)^{2}}, \quad \chi=\frac{(1-\eta)^{4}}{(1+2 \eta)^{2}} .
$$

Equations (E3)-(E6) define Wertheim-Thiele's exact solution of the PY integral equation for hard spheres [49, 50].

\section{Hyperspheres in five dimensions}

For a fluid of hyperspheres in $d=5(n=1)$ the definition of $G(s)$ is

$$
G(s)=\int_{0}^{\infty} \mathrm{d} r r(1+r s) g(r) e^{-s r} .
$$

Since $a_{0}=3$ and $\lambda=240$, Eq. (5.18) becomes in this case

$$
G(s)=\frac{e^{-s}}{s^{2}} \frac{3+a_{1} s+a_{2} s^{2}}{1+240 \eta\left[3 \phi_{5}(s)+a_{1} \phi_{4}(s)+a_{2} \phi_{3}(s)\right]} .
$$

Equation (5.26) yields $D_{1}=\widetilde{a}_{1}$ and $D_{3}+\gamma_{0} D_{1}=0$, where, according to Eq. (5.25), $\gamma_{0}=\widetilde{a}_{2}-D_{2}$. The physical solutions are

$$
a_{1}=\frac{3-3 \eta\left(1+10 a_{2}\right)}{1-6 \eta}
$$

$$
a_{2}=\frac{1+22 \eta+78 \eta^{2}+24 \eta^{3}+(1-\eta)(6 \eta-1) \xi}{20 \eta \xi^{2}},(\mathrm{E} 10)
$$

where $\xi \equiv \sqrt{1+18 \eta+6 \eta^{2}}$. The associated contact value and isothermal susceptibility are

$$
g\left(1^{+}\right)=\frac{\xi^{3}-1+33 \eta+87 \eta^{2}+6 \eta^{3}}{60 \eta(1-\eta)^{3}},
$$

$$
\chi=\frac{(1-\eta)^{2}}{\xi^{2}}\left[5\left(1+6 \eta+3 \eta^{2}\right)-2(2+3 \eta) \xi\right]
$$

It can be checked that Eq. (E8) yields the same structure factor as that given by the solution of the PY integral equation for $d=5$ obtained by a different method [8, 11].
[1] J.-P. Hansen and I. R. McDonald, Theory of Simple Liquids, 3rd. ed. (Academic Press, London, 2006).

[2] J. A. Barker and D. Henderson, Rev. Mod. Phys. 48, 587 (1976).

[3] Playing with Marbles: Theory and Simulation of HardSphere Fluids and Related Systems, edited by A. Mulero (Springer, in press).

[4] B. J. Alder and T. Wainwright, J. Chem. Phys. 33, 1439 (1960).

[5] W. G. Hoover and F. H. Ree, J. Chem. Phys. 49, 3609 (1968).

[6] J. P. J. Michels and N. J. Trappeniers, Phys. Lett. 104, 425 (1984).

[7] R. J. Speedy, J. Chem. Phys. 100, 6684 (1994).

[8] B. C. Freasier and D. J. Isbister, Mol. Phys. 42, 927 (1981).

[9] M. Luban and A. Baram, J. Chem. Phys. 76, 3233 (1982).

[10] C. G. Joslin, J. Chem. Phys. 77, 2701 (1982).

[11] E. Leutheusser, Physica 127A, 667 (1984).
[12] H. L. Frisch, N. Rivier, and D. Wyler, Phys. Rev. Lett. 54, 2061 (1985).

[13] E. Leutheusser, J. Chem. Phys. 84, 1050 (1986).

[14] Y. Rosenfeld, J. Chem. Phys. 87, 4865 (1987).

[15] D. Wyler, N. Rivier, and H. L. Frisch, Phys. Rev. A 36, 2422 (1987).

[16] M. Baus and J. L. Colot, Phys. Rev. A 36, 3912 (1987).

[17] Y. Song, E. A. Mason, and R. M. Stratt, J. Phys. Chem. 93, 6916 (1989).

[18] J. Amorós, J. R. Solana, and E. Villar, Phys. Chem. Liq. 19, 119 (1989).

[19] M. Luban and J. P. J. Michels, Phys. Rev. A 41, 6796 (1990).

[20] M. J. Maeso, J. R. Solana, J. Amorós, and E. Villar, Mater. Chem. Phys. 30, 39 (1991).

[21] D. J. González, L. E. González, and M. Silbert, Mol. Phys. 74, 613 (1991).

[22] J. G. Loeser, Z. Zhen, S. Kais, D. R. Herschbach, J. Chem. Phys. 95, 4525 (1991).

[23] H. L. Frisch and J. K. Percus, Phys. Rev. E 60, 2942 
(1999).

[24] E. Velasco, L. Mederos and G. Navascués, Mol. Phys. 97, 1273 (1999).

[25] M. Bishop, A. Masters, and J. H. R. Clarke, J. Chem. Phys. 110, 11449 (1999).

[26] G. Parisi and F. Slanina, Phys. Rev. E 62, 6554 (2000).

[27] S. B. Yuste, A. Santos, and M. López de Haro, Europhys. Lett. 52, 158 (2000).

[28] A. Santos, J. Chem. Phys. 112, 10680 (2000).

[29] M. González-Melchor, J. Alejandre, and M. López de Haro, J. Chem. Phys. 114, 4905 (2001).

[30] R. Finken, M. Schmidt, and H. Löwen, Phys. Rev. E 65, 016108 (2001).

[31] M. Robles, M. López de Haro, and A. Santos, J. Chem. Phys. 120, 9113 (2004); Erratum: 125 (2006).

[32] M. Bishop, A. Masters, and A. Yu. Vlasov, J. Chem. Phys. 121, 6884 (2004).

[33] N. Clisby and B. M. McCoy, J. Stat. Phys. 114, 1343 (2004); ibid. 114, 1361 (2004); ibid. 122, 15 (2006); Pramana 64, 775 (2005).

[34] I. Lyberg, J. Stat. Phys. 119, 747 (2005).

[35] A. Santos and M. López de Haro, Phys. Rev. E 72, 010501(R) (2005).

[36] L. Lue, J. Chem. Phys. 122, 044513 (2005).

[37] M. Bishop, P. A. Whitlock, and D. Klein, J. Chem. Phys. 122, 074508 (2005).

[38] M. Bishop, A. Masters, and A. Yu. Vlasov, J. Chem. Phys. 122, 154502 (2005).

[39] M. Bishop and P. A. Whitlock, J. Chem. Phys. 123, 014507 (2005).

[40] L. Lue and M. Bishop, Phys. Rev. E 74, 021201 (2006).

[41] M. Skoge, A. Donev, F. H. Stillinger, and S. Torquato, Phys. Rev. E 74, 041127 (2006).

[42] S. Torquato and F. H. Stillinger, Experimental Math. 15, 307 (2006).

[43] M. Robles, M. López de Haro, and A. Santos, J. Chem. Phys. 126, 016101 (2007).

[44] M. Bishop and P. A. Whitlock, J. Stat. Phys. 126, 299 (2007).

[45] P. A. Whitlock, M. Bishop, and J. L. Tiglias, J. Chem. Phys. 126, 224505 (2007).

[46] A. Scardicchio, F. H. Stillinger, and S. Torquato, e-print arXiv:0705.1482.

[47] G. Parisi, e-print arXiv:0710.0882

[48] J. I. Frenckel, Kinetic Theory of Liquids (Oxford University Press, London, 1946).

[49] M. S. Wertheim, Phys. Rev. Lett. 10, 321 (1963); J. Math. Phys. 5, 643 (1964).

[50] E. Thiele, J. Chem. Phys. 39, 474 (1963).

[51] S. Torquato, Random Heterogeneous Materials: Microstructure and Macroscopic Properties, (SpringerVerlag, New York, 2002).

[52] S. B. Yuste and A. Santos, Phys. Rev. A 43, 5418 (1991).

[53] M. López de Haro, S. B. Yuste, and A. Santos, "Alternative Approaches to the Equilibrium Properties of HardSphere Liquids," in Ref. [3]; e-print arXiv:0704.0157 [cond-mat.stat-mech].
[54] S. B. Yuste, M. López de Haro, and A. Santos, J. Chem. Phys. 108, 3683 (1998).

[55] S. B. Yuste and A. Santos, J. Stat. Phys. 72, 703 (1993).

[56] S. B. Yuste and A. Santos, Phys. Rev. E 48, 4599 (1993).

[57] A. Santos, S. B. Yuste, and M. López de Haro, J. Chem. Phys. 109, 6814 (1998).

[58] S. B. Yuste and A. Santos, J. Chem. Phys. 101, 2355 (1994).

[59] L. Acedo and A. Santos, J. Chem. Phys. 115, 2805 (2001).

[60] Al. Malijevský, S. B. Yuste, and A. Santos, Phys. Rev. E 76, 021504 (2007).

[61] Note that the diameter of the two intersecting hyperspheres considered in the evaluation of $y_{1}(r)$ is twice that of the true hard hyperspheres of the fluid.

[62] S. Bochner, Math. Zeit. 29, 730 (1929).

[63] H. L. Krall and O. Frink, Trans. Amer. Math. Soc. 65, 100 (1948).

[64] L. Carlitz, Duke Math. J. 24, 151 (1957).

[65] E. Grosswald, Bessel Polynomials (Springer-Verlag, New York, 1978).

[66] See also http://mathworld.wolfram.com/BesselPolynomial.html and http://en.wikipedia.org/wiki/Bessel_polynomials

[67] Y. Rosenfeld, Phys. Rev. A 32, 1834 (1985).

[68] S. Torquato, F. H. Stillinger, Phys. Rev. E 68, 041113 (2003).

[69] Of course, $\widehat{f}(k)$ can be expressed in terms of the Bessel function $J_{d / 2-1}$ using Eqs. (A3) and (A4).

[70] S. B. Yuste, M. López de Haro, and A. Santos, Phys. Rev. E 53, 4820 (1996).

[71] If one adopts the normalization condition $(5.15)$ instead of Eq. (5.14), one gets $b_{j}=\left[a_{0} /(d-2) ! !\right] \delta_{j, d}+$ $\lambda \eta \sum_{l=0}^{n+1}(-1)^{j-l} a_{l} /(j-l)$ !, with $0 \leq j \leq d$. For $j=d$, the above equation yields a linear relationship among all $a_{j}$. Instead of Eq. (5.18), the standard RFA can then be written in the equivalent form $G(s)=s^{-2} e^{-s} A_{n+1}(s) /\left[1+\lambda \eta s^{-1} \sum_{j=0}^{n+1} a_{j} \phi_{d-j-1}(s)\right]$. Notice that in that case $g\left(1^{+}\right)=a_{n+1}$.

[72] In fact, in the classical Debye-Hückel approximation for one-component plasmas the effective potential $V(r)$ is the solution to the Poisson equation $\nabla^{2} V(r)=-\epsilon_{0}^{-1} q \delta(\mathbf{r})+$ $k_{D}^{2} V(r)$, where $k_{D}$ is the Debye wavenumber. In Fourier space the solution is $\widehat{V}(k)=\left(q / \epsilon_{0}\right)\left(k^{2}+k_{D}^{2}\right)^{-1}$. Now, for $d=$ odd the inverse Fourier transform becomes $V(r)=\left[(2 \pi)^{(d-1) / 2} / 2 \epsilon_{0}\right] \theta_{n}\left(k_{D} r\right) r^{2-d} e^{-k_{D} r}$, where use has been made of Eq. (A12) and the residue theorem. It is in this sense in which we refer to $\theta_{n}(\kappa r) e^{-\kappa r} / r^{d-2}$ as a (generalized) Yukawa term.

[73] E. Waisman, Mol. Phys. 25, 45 (1973).

[74] http://www.research.att.com/ njas/lattices/density.html

[75] I. N. Sneddon, Fourier Transform (Dover Publications, New York, 1995).

[76] The ordinary Bessel polynomials are given by $y_{n}(t)=$ $t^{n} \theta_{n}(1 / t)$ (cf. Ref. [62]).

[77] M. Abramowitz and I. Stegun, Handbook of Mathematical Functions (Dover, New York, 1970). 